\title{
CONTACT PROBLEM OF CONDUCTING AND HEATED PUNCH ON A MULTIFIELD FOUNDATION
}

\author{
B. ROGOWSKI \\ Department of Mechanics of Materials \\ Lodz University of Technology \\ Al. Politechniki 6, 90-924 Lodz, POLAND \\ E-mail: bogdan.rogowski@p.lodz.pl
}

\begin{abstract}
The solution for a multifield material subjected to temperature loading in a circular region is presented in an explicit analytical form. The study concerns the steady - state thermal loading infinite region (heated embedded inclusion), half - space region and two - constituent magneto - electro - thermo - elastic material region. The new mono - harmonic potential functions, obtained by the author, are used in the analysis of punch problem. The more interested case in which the contact region is annular is analyzed. By using the methods of triple integral equations and series solution technique the solution for an indentured multifield substrate over an annular contact region is given. The sensitivity analysis of obtained indentation parameters shows some interesting points. In particular, it shows that the increasing of the applied electric and magnetic potentials reduces the indentation depth in multifield materials.
\end{abstract}

Key words: magneto - electro - thermo - elastic medium, conducting and heated punch, contact problem, exact solution.

\section{Introduction}

Solutions for various cases of contact problems can be found in the books by Gladwell (1980), Johnson (1985), Hills et al. (1993), Raous et al. (1995) and Rogowski (2006; 2006). However, in these books elastic fields themselves are considered and distinctive mathematical methods are used and elaborated. However, there is no parallel work in the domain of magneto - electro - thermo - elasticity. In the recent paper by Chen et al. (2010) the authors obtained the coupled fields for indentation of a multiferroic composite half - space for three common indenters: flat - ended, conical and spherical. The complete contact problem is considered under the assumption of circular contact region. Various important discussions related to indentation of piezoelectric materials, e.g., on the piezoresponse force microscopy (Kalinin et al., 2004) can be directly borrowed and applied to piezomagnetic materials. It is reasonable to assume that the extension of the findings to multifield composite materials is valid. The effective solution to the contact problem of multifield foundation and truncated conical punch or punch with a concave base was obtained and published recently by the author (Rogowski and Kaliński, 2012; Rogowski, 2012). In this paper, five potential mono - harmonic functions, obtained by the author, are utilized to solve the punch problem in which the contact region is always annular. The outer circumference of the annulus coincides with the edge of the punch. The inner circumference will shrink with an increasing load. The inner radius is not known a priori and is obtained from the conditions of equal thermal displacement and indentation mechanical depth of the punch on this circumference. On this boundary the phenomenon of adhesive contact is observed. The problem is solved by triple integral equations technique. The relationships between the force, electric charge, magnetic flux, temperature, indentation depth of the punch and electric and magnetic potentials on the boundary are derived. 


\section{Axisymmetric solutions of a multifield body}

The generalized multifield solution considered here is independent of the variable $\theta$ in the cylindrical coordinate system $(r, \theta, z)$. Therefore, the mechanical displacement $u_{r}$ and $u_{z}$, the electric potential $\phi$ and magnetic potential $\psi$, the mechanical stresses $\sigma_{r}, \sigma_{\theta}, \sigma_{z}$ and $\sigma_{r z}$, the electric displacements $D_{r}$ and $D_{z}$, the magnetic inductions $B_{r}$ and $B_{z}$ can be generally expressed as (Rogowski, 2014).

$$
\begin{aligned}
& u_{r}(r, z)=\sum_{i=0}^{4} a_{1 i} \lambda_{i} \frac{\partial \varphi_{i}}{\partial r}, \quad u_{z}(r, z)=\sum_{i=0}^{4} \frac{\partial \varphi_{i}}{\partial z_{i}}, \\
& \phi(r, z)=-\sum_{i=0}^{4} a_{3 i} \frac{\partial \phi_{i}}{\partial z_{i}}, \quad \psi(r, z)=-\sum_{i=0}^{4} a_{4 i} \frac{\partial \phi_{i}}{\partial z_{i}}, \\
& \sigma_{r}=-\sum_{i=0}^{4} a_{5 i} \lambda_{i} \frac{\partial^{2} \phi_{i}}{\partial z_{i}^{2}}-\left(c_{11}-c_{12}\right) \frac{u_{r}}{r}-\beta_{1} T \\
& \sigma_{\theta}=-\sum_{i=0}^{4} a_{5 i} \lambda_{i} \frac{\partial^{2} \phi_{i}}{\partial z_{i}^{2}}-\left(c_{11}-c_{12}\right) \frac{\partial u_{r}}{\partial r}-\beta_{1} T \\
& \sigma_{z}=\sum_{i=0}^{4} \frac{a_{5 i}}{\lambda_{i}} \frac{\partial^{2} \varphi_{i}}{\partial z_{i}^{2}}+\lambda_{0}^{-2} \beta_{1} T, \quad \quad \sigma_{r z}=\sum_{i=0}^{4} a_{5 i} \frac{\partial^{2} \varphi_{i}}{\partial r \partial z_{i}}+\lambda_{0}^{-1} \beta_{1} a_{00} \frac{\partial^{2} \varphi_{0}}{\partial r \partial z_{0}} \\
& D_{r}=\sum_{i=0}^{4} a_{6 i} \lambda_{i}^{2} \frac{\partial^{2} \varphi_{i}}{\partial r \partial z_{i}}, \quad D_{z}=\sum_{i=0}^{4} a_{6 i} \lambda_{i} \frac{\partial^{2} \varphi_{i}}{\partial z_{i}^{2}} \\
& B_{r}=\sum_{i=0}^{4} a_{7 i} \lambda_{i}^{2} \frac{\partial^{2} \phi_{i}}{\partial r \partial z_{i}}, \quad B_{z}=\sum_{i=0}^{4} a_{7 i} \lambda_{i} \frac{\partial^{2} \phi_{i}}{\partial z_{i}^{2}}
\end{aligned}
$$

In addition

$$
E_{r}=-\frac{\partial \varphi}{\partial r}, \quad E_{z}=-\frac{\partial \varphi}{\partial z}, \quad H_{r}=-\frac{\partial \psi}{\partial r}, \quad H_{z}=-\frac{\partial \psi}{\partial z},
$$

are the components of electric and magnetic field vectors.

In the fundamental solution $\varphi_{i}\left(r, z_{i}\right)$ are the harmonic functions of the variables $r$ and $z_{i}=\lambda_{i} z$, that is

$$
\frac{\partial^{2} \varphi_{i}}{\partial r^{2}}+\frac{1}{r} \frac{\partial \varphi_{i}}{\partial r}+\frac{\partial^{2} \varphi_{i}}{\partial z_{i}^{2}}=0, \quad i=0,1,2,3,4
$$

The temperature in a steady - state and uncoupled thermoelastic problem is governed by 


$$
\lambda_{r}\left(\frac{\partial^{2} T}{\partial r^{2}}+\frac{1}{r} \frac{\partial}{\partial r}\right)+\lambda_{z} \frac{\partial^{2} T}{\partial z^{2}}=0
$$

where $\lambda_{r}, \lambda_{z}$ are the heat conduction coefficients in unit $\mathrm{W} / \mathrm{Km}$ and $T$ is described by $\varphi_{0}\left(r, z_{0}\right)$ as follows

$$
T\left(r, z_{0}\right)=a_{00} \frac{\partial^{2} \varphi_{0}}{\partial z_{0}^{2}}, \quad z_{0}=\lambda_{0} z, \quad \lambda_{0}=\sqrt{\frac{\lambda_{r}}{\lambda_{z}}}
$$

The quantities $\lambda_{i}(i=1,2,3,4)$ are eigenvalues of multifield material defined as the roots of equation

$$
a \lambda^{8}+b \lambda^{6}+c \lambda^{4}+d \lambda^{2}+e=0
$$

where $a, b, c, d$ and $e$ are given in the Appendix A by Eqs A1. The roots of Eq.(2.6) are presented by formulae A4. The other material parameters are given by

$$
\begin{aligned}
\left\{\begin{array}{l}
a_{1 i} \\
a_{3 i} \\
a_{4 i}
\end{array}\right\}= & {\left[\begin{array}{ccc}
\lambda_{i}^{2}\left(e_{31}+e_{15}\right) & -\left(\varepsilon_{33} \lambda_{i}^{2}-\varepsilon_{11}\right) & -\left(d_{33} \lambda_{i}^{2}-d_{11}\right) \\
\lambda_{i}^{2}\left(q_{31}+q_{15}\right) & -\left(d_{33} \lambda_{i}^{2}-d_{11}\right) & -\left(\mu_{33} \lambda_{i}^{2}-\mu_{11}\right) \\
c_{11}+c_{13} \lambda_{i}^{2} & e_{31}+e_{33} \lambda_{i}^{2} & q_{31}+q_{33} \lambda_{i}^{2}
\end{array}\right]^{-1}\left\{\begin{array}{r}
e_{33} \lambda_{i}^{2}-e_{15}+p_{3} \lambda_{i} a_{00} \delta_{i 0} \\
q_{33} \lambda_{i}^{2}-q_{15}+\gamma_{3} \lambda_{i} a_{00} \delta_{i 0} \\
c_{33} \lambda_{i}^{2}+c_{13}-\left(\beta_{3}+\beta_{1} \lambda_{i}^{-2}\right) a_{00} \lambda_{i} \delta_{i 0}
\end{array}\right\} } \\
& a_{5 i}=c_{11} a_{1 i}-c_{13}+e_{31} a_{3 i}+q_{31} a_{4 i} \\
& \lambda_{i}^{2} a_{6 i}=\lambda_{i}^{2} e_{15} a_{1 i}+e_{15}+\varepsilon_{11} a_{3 i}+d_{11} a_{4 i}, \\
& \lambda_{i}^{2} a_{7 i}=\lambda_{i}^{2} q_{15} a_{1 i}+q_{15}+\mu_{11} a_{4 i}+d_{11} a_{3 i} \\
& a_{00}=\frac{a \lambda_{0}^{8}+b \lambda_{0}^{6}+c \lambda_{0}^{4}+d \lambda_{0}^{2}+e}{a_{2} \lambda_{0}^{6}+b_{2} \lambda_{0}^{4}+c_{2} \lambda_{0}^{2}+d_{2}}
\end{aligned}
$$

where in the last equation the denominator is exactly given by the determinant of the matrix in Eq.(2.7). The unit of $\left[a_{00}\right]=10^{6} \mathrm{~K}$. In addition, the thermal moduli $\beta_{1}, \beta_{3}$, pyroelectric parameter $p_{3}$ and pyromagnetic parameter $\gamma_{3}$ are defined as follows

$$
\begin{aligned}
& \beta_{1}=\left(c_{11}+c_{12}\right) \alpha_{r}+c_{13} \alpha_{z}, \quad \beta_{3}=2 c_{13} \alpha_{r}+c_{33} \alpha_{z}, \\
& p_{3}=2 e_{11} \alpha_{r}+e_{33} \alpha_{z}, \quad \gamma_{3}=2 q_{31} \alpha_{r}+q_{33} \alpha_{z}
\end{aligned}
$$

where $\alpha_{r}$ and $\alpha_{z}$ are the coefficients of thermal expansion $[\alpha]=10^{6} 1 / K$. The units of these parameters are

$$
\left[\beta_{1}, \beta_{3}\right]=10^{5} \mathrm{~N} / \mathrm{m}^{2} \mathrm{~K}, \quad\left[p_{3}\right]=10^{-6} \mathrm{C} / \mathrm{m}^{2} \mathrm{~K}, \quad\left[\gamma_{3}\right]=10^{-4} \mathrm{~N} / \mathrm{AmK}
$$


The multifield material is characterized by twenty one material constants. There are: five elastic constants $c_{11}, c_{12}, c_{13}, c_{33}, c_{44}$ (in units GPa), three piezoelectric constants $e_{31}, e_{15}, e_{33}$ (in units $C / m^{2}$ ), three piezomagnetic constants $q_{31}, q_{15}, q_{33}$ (in units $N / A m$ ), two magneto - electric constants $d_{11}, d_{33}$ (in units $10^{-9} \mathrm{C} / \mathrm{Am}$ ), two dielectric permittivities $\varepsilon_{11}, \varepsilon_{33}$ (in units $10^{-10} \mathrm{C} / \mathrm{Vm}$ ), two magnetic permeabilities $\mu_{11}, \mu_{33}$ (in units $10^{-6} N / A^{2}$ ), two coefficients of thermal expansion $\alpha_{r}, \alpha_{z}$ (in units $10^{-6} 1 / K$ ) and two coefficients of heat conduction $\lambda_{r}, \lambda_{z}$ (in unit $W / K m$ ). Any solution other than explicit analytical ones is impractical in the context of multifield material.

\section{The temperature changes}

We seek the harmonic function $\varphi_{0}\left(r, z_{0}\right)$ in the form

$$
\varphi_{0}\left(r, z_{0}\right)=\frac{1}{a_{00}} \int_{0}^{a}\left[\frac{1}{2} \xi_{0} \eta_{0} \ln \frac{1+\eta_{0}}{1-\eta_{0}}-\xi_{0}-\tan ^{-1} \frac{1}{\xi_{0}}\right] x \varphi(x) d x
$$

where $\varphi(x)$ to be determined from the thermal field boundary conditions.

The spheroidal coordinates $\xi_{i}$ and $\eta_{i}$ are related to cylindrical coordinates $r, \lambda_{i} z$ by equations

$$
r^{2}=x^{2}\left(1+\xi_{i}^{2}\right)\left(1-\eta_{i}^{2}\right), \quad \lambda_{i} z=x \xi_{i} \eta_{i}, \quad \xi_{i} \geq 0, \quad\left|\eta_{i}\right| \leq 1
$$

and are associated with $\lambda_{i}$ (here $i=0$ ) and with $0 \leq x \leq a$. The derivatives may be easily calculated, that is

$$
\begin{aligned}
& \frac{\partial \phi_{0}}{\partial r}=-\frac{1}{a_{00}} \frac{1}{r} \int_{0}^{a} x \xi_{0}\left(1-\eta_{0}\right) \phi(x) d x, \\
& \frac{\partial \varphi_{0}}{\partial z}=\frac{\lambda_{0}}{2 a_{00}} \int_{0}^{a} \ln \frac{1+\eta_{0}}{1-\eta_{0}} \varphi(x) d x, \\
& \frac{\partial^{2} \phi_{0}}{\partial r \partial z}=\frac{\lambda_{0}}{a_{00}} \frac{1}{r} \int_{0}^{a}\left[1-\frac{\partial}{\partial x}\left(x \eta_{0}\right)\right] \phi(x) d x, \\
& \frac{\partial^{2} \phi_{0}}{\partial z^{2}}=\frac{\lambda_{0}^{2}}{a_{00}} \int_{0}^{a} \frac{\xi_{0}}{\xi_{0}^{2}+\eta_{0}^{2}} \frac{\phi(x)}{x} d x, \\
& \frac{\partial^{2} \phi_{0}}{\partial r^{2}}=\frac{1}{a_{00}} \int_{0}^{a}\left[\frac{x}{r^{2}} \xi_{0}\left(1-\eta_{0}\right)-\frac{\xi_{0}}{x\left(\xi_{0}^{2}+\eta_{0}^{2}\right)}\right] \phi(x) d x, \\
& \frac{\partial^{3} \varphi_{0}}{\partial z^{3}}=-\frac{\lambda_{0}^{3}}{a_{00}} \int_{0}^{a} \frac{\partial}{\partial x}\left(\frac{\eta_{0}}{x\left(\xi_{0}^{2}+\eta_{0}^{2}\right)}\right) \varphi(x) d x .
\end{aligned}
$$


The quantities $Q=-\lambda_{z} \partial T / \partial z, \phi, \psi$ and $u_{z}$ associated with the thermoelastic potential $\phi_{0}$ vanish for $z=0, r \geq a$, since $\eta=0$ for $z=0, r \geq a$.

The temperature and heat flux are

$$
T(r, z)=\int_{0}^{a} \frac{\xi_{0}}{\xi_{0}^{2}+\eta_{0}^{2}} \frac{\phi(x)}{x} d x, \quad Q(r, z)=\sqrt{\lambda_{r} \lambda_{z}} \int_{0}^{a} \frac{\partial}{\partial x}\left(\frac{\eta_{0}}{x\left(\xi_{0}^{2}+\eta_{0}^{2}\right)}\right) \phi(x) d x .
$$

The condition for the prescribed temperature $T(r, 0)=f(r)$ for $r \leq a$ gives

$$
\int_{0}^{r} \frac{\varphi(x) d x}{\sqrt{r^{2}-x^{2}}}=f(r)
$$

This is Abel's integral equation with the solution

$$
\phi(x)=\frac{2}{\pi} \frac{d}{d x} \int_{0}^{x} \frac{r f(r) d r}{\sqrt{x^{2}-r^{2}}} .
$$

If we assume the distribution of temperature to be cylindrical constant or revolution conical or revolution parabolic, that is if

$$
f(r)=T_{0}\left(1,1-\frac{r}{a}, 1-\left(\frac{r}{a}\right)^{2}\right), \quad r \leq a
$$

the solution $\phi(x)$ will be

$$
\phi(x)=\frac{2}{\pi} T_{0}\left(1,1-\frac{\pi x}{2 a}, 1-2\left(\frac{x}{a}\right)^{2}\right)
$$

respectively.
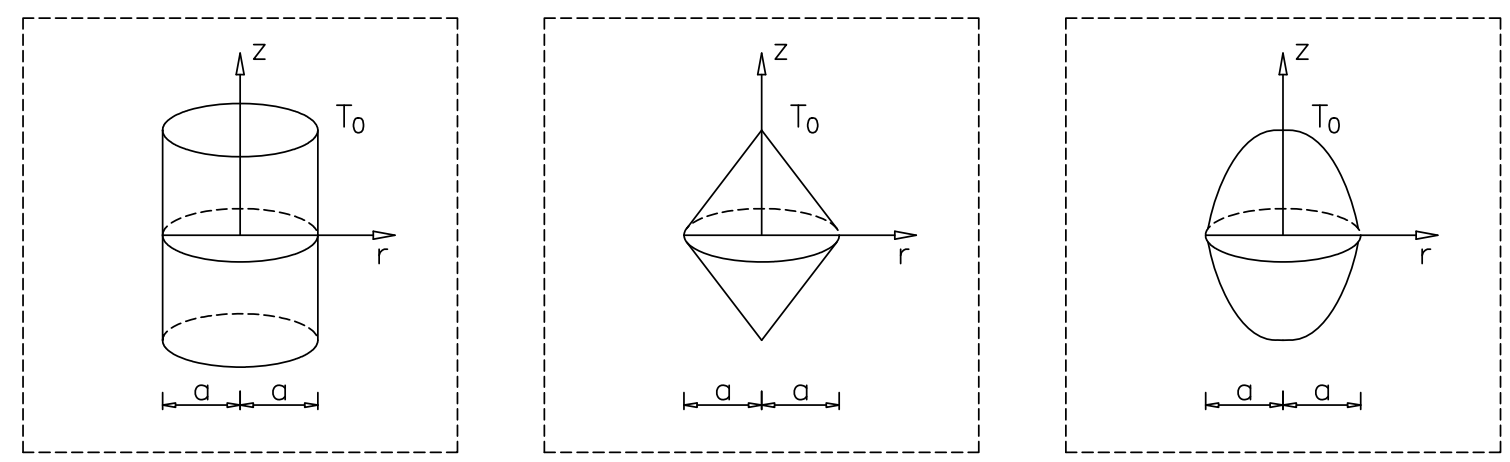

Fig.1. Multifield material with temperature change in the circular region $r \leq a$ as to be constant or revolution conical and revolution parabolic. 
For constant temperature $T_{0}$ we have (here $\xi_{0}$ and $\eta_{0}$ are for $x=a$ )

$$
\begin{aligned}
& \phi_{0}(r, z)=\frac{2}{\pi} T_{0} a^{2} a_{00}\left[\frac{\lambda_{0} z}{a}\left(\frac{1}{2} \ln \frac{1+\eta_{0}}{1-\eta_{0}}-\frac{1}{\eta_{0}}\right)-\frac{1}{2}\left(\tan ^{-1} \frac{1}{\xi_{0}}-\tan ^{-1} \frac{\lambda_{0} z}{a}\right)+\right. \\
& \left.-\frac{\lambda_{0}^{2} z^{2}}{a^{2}}\left(\tan ^{-1} \xi_{0}+\frac{1}{\xi_{0}}-\tan ^{-1} \frac{\lambda_{0} z}{a}-\frac{a}{\lambda_{0} z}\right)-\frac{r^{2}}{4 a^{2}}\left(\tan ^{-1} \frac{1}{\xi_{0}}-\frac{\xi_{0}}{1+\xi_{0}^{2}}\right)\right] \text {, } \\
& u_{r}^{(0)}(r, z)=-\frac{T_{0} r a_{10} \lambda_{0}}{\pi a_{00}}\left(\frac{\pi}{2}-\tan ^{-1} \xi_{0}+\frac{\xi_{0}}{1+\xi_{0}} \frac{1-\eta_{0}}{1+\eta_{0}}\right), \\
& {\left[\begin{array}{c}
u_{z}^{(0)}(r, z) \\
\phi^{(0)}(r, z) \\
\psi^{(0)}(r, z)
\end{array}\right]=\frac{2 T_{0} a}{\pi}\left[\begin{array}{c}
1 \\
-a_{30} \\
-a_{40}
\end{array}\right] \frac{1}{a_{00}}\left[\frac{1}{2} \ln \frac{1+\eta_{0}}{1-\eta_{0}}-\eta_{0}\left(1-\xi_{0} \tan ^{-1} \frac{1}{\xi_{0}}\right)\right],} \\
& {\left[\begin{array}{c}
\sigma_{z r}^{(0)}(r, z) \\
E_{r}^{(0)}(r, z) \\
H_{r}^{(0)}(r, z) \\
D_{r}^{(0)}(r, z) \\
B_{r}^{(0)}(r, z)
\end{array}\right]=\frac{2 T_{0}}{\pi a_{00}} \frac{a}{r}\left[\begin{array}{c}
a_{50}+\frac{\beta_{1}}{\lambda_{0}} a_{00} \\
a_{30} \\
a_{40} \\
a_{60} \lambda_{0}^{2} \\
a_{70} \lambda_{0}^{2}
\end{array}\right]\left(1-\eta_{0}\right)} \\
& {\left[\begin{array}{c}
\sigma_{z z}^{(0)}(r, z) \\
E_{z}^{(0)}(r, z) \\
H_{z}^{(0)}(r, z) \\
D_{z}^{(0)}(r, z) \\
B_{z}^{(0)}(r, z)
\end{array}\right]=\frac{2 T_{0}}{\pi a_{00}}\left[\begin{array}{c}
\left(a_{50}+\frac{\beta_{1}}{\lambda_{0}} a_{00}\right) \frac{1}{\lambda_{0}} \\
a_{30} \lambda_{0} \\
a_{40} \lambda_{0} \\
a_{60} \lambda_{0} \\
a_{70} \lambda_{0}
\end{array}\right]\left(\frac{\pi}{2}-\tan ^{-1} \xi_{0}\right),} \\
& {\left[\begin{array}{l}
\sigma_{r r}^{(0)}(r, z) \\
\sigma_{\theta \theta}^{(0)}(r, z)
\end{array}\right]=-\frac{2 T_{0}}{\pi}\left(\frac{a_{50}}{a_{00}} \lambda_{0}+\beta_{1}\right)\left(\frac{\pi}{2}-\tan ^{-1} \xi_{0}\right)-\left[\begin{array}{c}
\left(c_{11}-c_{12}\right) \frac{u_{r}^{(0)}(r, z)}{r} \\
\left(c_{11}-c_{12}\right) \frac{\partial u_{r}^{(0)}(r, z)}{\partial r}
\end{array}\right],} \\
& T(r, z)=T_{0}\left(1-\frac{2}{\pi} \tan ^{-1} \xi_{0}\right)
\end{aligned}
$$

where 


$$
\xi_{0}=\frac{1}{2} \sqrt{\left(\sqrt{\frac{\lambda_{0}^{2} z^{2}}{a^{2}}+\left(\frac{r}{a}+1\right)^{2}}+\sqrt{\frac{\lambda_{0}^{2} z^{2}}{a^{2}}+\left(\frac{r}{a}-1\right)^{2}}\right)}, \quad \eta_{0}=\frac{\lambda_{0} z}{a \xi_{0}} .
$$

The fundamental equations are single - direction coupling such that the thermal loading can change electro - magneto - elastic fields, the mechanical, electric and magnetic loadings cannot change the thermal field. This is a theory of uncoupled thermoelasticity of multifield material where the temperature field is independent of the electric displacement, and in addition, the inertial terms can be neglected.

To satisfy the zero - shear condition at $z=0$, we find field defined by harmonic potential (Hankel integral)

$$
\phi_{i}(r, z)=\int_{0}^{\infty} \xi^{-1} A_{i}(\xi) e^{-\lambda_{i} \xi z} J_{0}(\xi r) d \xi, \quad i=1,2,3,4,
$$

in which

$$
m_{2}^{*} A_{i}(\xi)=-\frac{2}{\pi} T_{0} \tilde{\beta} \frac{\sin \xi a}{\xi^{2}} d_{i}^{*}, \quad \tilde{\beta}=\frac{a_{50}}{a_{00}}+\frac{\beta_{1}}{\lambda_{0}}
$$

where $m_{2}^{*}$ and $d_{i}^{*}$ are defined in the Appendix A by Eqs (A5).

Since

$$
\sum a_{3 i} d_{i}^{*}=\sum a_{4 i} d_{i}^{*}=\sum \frac{a_{5 i} d_{i}^{*}}{\lambda_{i}}=0, \quad m_{2}^{*}=\sum a_{5 i} d_{i}^{*}, \quad \sum \equiv \sum_{i=1}^{i=4},
$$

the solution satisfies the boundary conditions at $z=0, r \geq 0$

$$
\phi=\psi=\sigma_{z}=0, \quad \sigma_{z r}=-\frac{2}{\pi} \tilde{\beta} T_{0}\left(\frac{a}{r}-\sqrt{\frac{a^{2}}{r^{2}}-1 H}\left(\frac{a}{r}-1\right)\right)
$$

where $H(\bullet)$ denotes Heaviside's unit function.

The full field in this case is given in analytical form, that is

$$
\begin{aligned}
& u_{r}(r, z)=\frac{1}{\pi} T_{0} \tilde{\beta} \frac{r}{m_{2}^{*}} \sum_{i=1}^{4} a_{1 i} \lambda_{i} d_{i}^{*}\left(\frac{\pi}{2}-\tan ^{-1} \xi_{i}+\frac{\xi_{i}}{1+\xi_{i}^{2}} \frac{1-\eta_{i}}{1+\eta_{i}}\right), \\
& {\left[\begin{array}{l}
u_{z}(r, z) \\
-\varphi(r, z) \\
-\psi(r, z)
\end{array}\right]=-\frac{2}{\pi} T_{0} \tilde{\beta} \frac{a}{m_{2}^{*}} \sum_{i=1}^{4}\left[\begin{array}{c}
d_{i}^{*} \\
a_{3 i} d_{i}^{*} \\
a_{4 i} d_{i}^{*}
\end{array}\right]\left[\frac{1}{2} \ln \frac{1+\eta_{i}}{1-\eta_{i}}-\eta_{i}\left(1-\xi_{i}\left(\frac{\pi}{2}-\tan ^{-1} \frac{1}{\xi_{i}}\right)\right),\right.}
\end{aligned}
$$




$$
\begin{aligned}
& {\left[\begin{array}{c}
\sigma_{z r}(r, z) \\
E_{r}(r, z) \\
H_{r}(r, z) \\
D_{r}(r, z) \\
B_{r}(r, z)
\end{array}\right]=-\frac{2}{\pi} T_{0} \tilde{\beta} \frac{a}{r} \frac{1}{m_{2}^{*}} \sum_{i=1}^{4}\left[\begin{array}{c}
a_{5 i} d_{i}^{*} \\
a_{3 i} d_{i}^{*} \\
a_{4 i} d_{i}^{*} \\
a_{6 i} \lambda_{i}^{2} d_{i}^{*} \\
a_{7 i} \lambda_{i}^{2} d_{i}^{*}
\end{array}\right]\left(1-\eta_{i}\right),} \\
& {\left[\begin{array}{l}
\sigma_{z z}(r, z) \\
E_{z}(r, z) \\
H_{z}(r, z) \\
D_{z}(r, z) \\
B_{z}(r, z)
\end{array}\right]=-\frac{2}{\pi} T_{0} \tilde{\beta} \frac{1}{m_{2}^{*}} \sum_{i=1}^{4}\left[\begin{array}{c}
a_{5 i} \\
\lambda_{i} \\
a_{3 i} d_{i}^{*} \lambda_{i} \\
a_{4 i} d_{i}^{*} \lambda_{i} \\
a_{6 i} d_{i}^{*} \lambda_{i} \\
a_{7 i} d_{i}^{*} \lambda_{i}
\end{array}\right]\left(\frac{\pi}{2}-\tan ^{-1} \xi_{i}\right),} \\
& {\left[\begin{array}{c}
\sigma_{r r}(r, z) \\
\sigma_{\theta \theta}(r, z)
\end{array}\right]=\frac{2}{\pi} T_{0} \tilde{\beta} \frac{1}{m_{2}^{*}} \sum_{i=1}^{4} a_{5 i} \lambda_{i} d_{i}^{*}\left(\frac{\pi}{2}-\tan ^{-1} \xi_{i}\right)-\left[\begin{array}{l}
\left(c_{11}-c_{12}\right) \frac{u_{r}(r, z)}{r} \\
\left(c_{11}-c_{12}\right) \frac{\partial u_{r}(r, z)}{\partial r}
\end{array}\right] .}
\end{aligned}
$$

The nonsingular thermoelastic coupled field can be obtained by superimposing the two parts as given by Eqs (3.9) and (3.15). For a multifield material and embedded interior thin heated inclusion subjected to temperature $T_{0}$ at the contact surface the normal and shear stresses, electric and magnetic potentials and electric displacement and magnetic induction in the axial direction are as follow

$$
\begin{aligned}
& \sigma_{z z}(r, z)=T_{0} \tilde{\beta}\left[\frac{1}{\lambda_{0}}\left(1-\frac{2}{\pi} \tan ^{-1} \xi_{0}\right)+\frac{2}{\pi} \frac{1}{m_{2}^{*}} \sum_{i=1}^{4} \frac{a_{5 i}}{\lambda_{i}} d_{i}^{*} \tan ^{-1} \xi_{i}\right], \\
& \sigma_{z r}(r, z)=T_{0} \tilde{\beta}\left(\eta_{0}-\frac{1}{m_{2}^{*}} \sum_{i=1}^{4} a_{5 i} d_{i}^{*} \eta_{i}\right), \\
& u_{z}(r, z)=\frac{2 T_{0} a}{\pi}\left[\frac{1}{a_{00}} \ln \frac{1+\eta_{0}}{1-\eta_{0}}-\eta_{0}\left(1-\xi_{0} \tan ^{-1} \frac{1}{\xi_{0}}\right)+\right. \\
& \left.+\frac{\tilde{\beta}}{m_{2}^{*}} \sum_{i=1}^{4} d_{i}^{*}\left(\frac{1}{2} \ln \frac{1+\eta_{i}}{1-\eta_{i}}-\eta_{i}\left(1-\xi_{i} \tan ^{-1} \frac{1}{\xi_{i}}\right)\right)\right] \text {, } \\
& \varphi(r, z)=-\frac{2 T_{0} a}{\pi}\left[\frac{a_{30}}{a_{00}}\left(\frac{1}{2} \ln \frac{1+\eta_{0}}{1-\eta_{0}}-\eta_{0}\left(1-\xi_{0} \tan ^{-1} \frac{1}{\xi_{0}}\right)\right)+\right. \\
& \left.+\frac{\tilde{\beta}}{m_{2}^{*}} \sum_{i=1}^{4} a_{3 i} d_{i}^{*}\left(\frac{1}{2} \ln \frac{1+\eta_{i}}{1-\eta_{i}}-\eta_{i}\left(1-\xi_{i} \tan ^{-1} \frac{1}{\xi_{i}}\right)\right)\right] \text {, }
\end{aligned}
$$




$$
\begin{aligned}
& \psi(r, z)=-\frac{2 T_{0} a}{\pi}\left[\frac{a_{40}}{a_{00}}\left(\frac{1}{2} \ln \frac{1+\eta_{0}}{1-\eta_{0}}-\eta_{0}\left(1-\xi_{0} \tan ^{-1} \frac{1}{\xi_{0}}\right)\right)+\right. \\
& \left.+\frac{\tilde{\beta}}{m_{2}^{*}} \sum_{i=1}^{4} a_{4 i} d_{i}^{*}\left(\frac{1}{2} \ln \frac{1+\eta_{i}}{1-\eta_{i}}-\eta_{i}\left(1-\xi_{i} \tan ^{-1} \frac{1}{\xi_{i}}\right)\right)\right], \\
& D_{z}(r, z)=T_{0}\left[\left(p_{3}+p_{3}^{*}\right)\left(1-\frac{2}{\pi} \tan ^{-1} \xi_{0}\right)-\frac{\tilde{\beta}}{m_{2}^{*}} \sum_{i=1}^{4} a_{6 i} \lambda_{i} d_{i}^{*}\left(1-\frac{2}{\pi} \tan ^{-1} \xi_{i}\right)\right], \\
& B_{z}(r, z)=T_{0}\left[\left(\gamma_{3}+\gamma_{3}^{*}\right)\left(1-\frac{2}{\pi} \tan ^{-1} \xi_{0}\right)-\frac{\tilde{\beta}}{m_{2}^{*}} \sum_{i=1}^{4} a_{7 i} \lambda_{i} d_{i}^{*}\left(1-\frac{2}{\pi} \tan ^{-1} \xi_{i}\right)\right]
\end{aligned}
$$

where

$$
\begin{aligned}
& p_{3}^{*}=\frac{a_{60}^{*} \lambda_{0}}{a_{00}}, \quad \gamma_{3}^{*}=\frac{a_{70}^{*} \lambda_{0}}{a_{00}}, \quad \tilde{\beta}=\frac{\beta_{1}}{\lambda_{0}}+\beta_{1}^{*}, \quad \beta_{1}^{*}=\frac{a_{50}}{a_{00}}, \\
& a_{60}^{*}=e_{33}-e_{31} a_{10}+\varepsilon_{33} a_{30}+d_{33} a_{40}, \\
& a_{70}^{*}=q_{33}-q_{31} a_{10}+d_{33} a_{30}+\mu_{33} a_{40} .
\end{aligned}
$$

The units of thermal moduli $\beta_{1}$ and $\beta_{1}^{*}$, pyroelectric and pyromagnetic constants $p_{3}$ and $p_{3}^{*}$, and $\gamma_{3}$ and $\gamma_{3}^{*}$ are

$$
\left[\beta_{1}, \beta_{1}^{*}\right]=10^{5} \mathrm{~N} /\left(\mathrm{m}^{2} \mathrm{~K}\right),\left[\mathrm{p}_{3}, p_{3}^{*}\right]=10^{-6} \mathrm{C} /\left(\mathrm{m}^{2} \mathrm{~K}\right), \quad\left[\gamma_{3}, \gamma_{3}^{*}\right]=10^{-4} \mathrm{~N} /(\mathrm{AmK})
$$

On the plane $z=0$ we have

$$
\begin{aligned}
& {\left[\begin{array}{c}
\sigma_{z z} \\
D_{z} \\
B_{z}
\end{array}\right]=\left[\begin{array}{c}
\sigma_{T} \\
D_{T} \\
B_{T}
\end{array}\right]=T_{0}\left[\begin{array}{c}
\tilde{\beta} / \lambda_{0} \\
p \\
\gamma
\end{array}\right]\left[1-\left(1-\frac{2}{\pi} \arcsin \left(\frac{a}{r}\right) H\left(1-\frac{a}{r}\right)\right)\right],} \\
& \sigma_{z r} \equiv 0 \\
& {\left[\begin{array}{c}
u_{z} \\
\varphi \\
\psi
\end{array}\right]=\left[\begin{array}{c}
u_{T} \\
\varphi_{T} \\
\psi_{T}
\end{array}\right]=\frac{2}{\pi} T_{0} a\left[\begin{array}{c}
\tilde{\alpha} \\
-\tilde{e} \\
-\tilde{q}
\end{array}\right]\left\{\begin{array}{l}
\ln \left(\frac{a}{r}+\sqrt{\frac{a^{2}}{r^{2}}-1}\right)-\sqrt{1-\frac{r^{2}}{a^{2}}}, \quad r \leq a \\
0 \quad, \quad r \geq a
\end{array}\right.}
\end{aligned}
$$

where 


$$
\begin{aligned}
& p=p_{3}+p_{3}^{*}-\frac{\tilde{\beta}}{m_{2}^{*}} \sum_{i=1}^{4} a_{6 i} \lambda_{i} d_{i}^{*}=p_{3}+p_{3}^{*}-p_{3}^{* *}, \quad\left[10^{-6} \mathrm{C} / \mathrm{m}^{2} \mathrm{~K}\right] \\
& \gamma=\gamma_{3}+\gamma_{3}^{*}-\frac{\beta}{m_{2}^{*}} \sum_{i=1}^{4} a_{7 i} \lambda_{i} d_{i}^{*}=\gamma_{3}+\gamma_{3}^{*}-\gamma_{3}^{* *}, \quad\left[10^{-4} \mathrm{~N} / \mathrm{m}^{2} \mathrm{~K}\right] \\
& \tilde{\alpha}=\frac{1}{a_{00}}+\frac{\tilde{\beta}}{m_{2}^{*}} \sum_{i=1}^{4} d_{i}^{*}, \quad\left[10^{-6} 1 / \mathrm{K}\right], \\
& \tilde{e}=\frac{a_{30}}{a_{00}}+\frac{\tilde{\beta}}{m_{2}^{*}} \sum_{i=1}^{4} a_{3 i} d_{i}^{*}, \quad\left[10^{-6} \mathrm{C} / \mathrm{mK}\right], \\
& \tilde{q}=\frac{a_{40}}{a_{00}}+\frac{\tilde{\beta}}{m_{2}^{*}} \sum_{i=1}^{4} a_{4 i} d_{i}^{*}, \quad\left[10^{-4} \mathrm{~A} / \mathrm{mK}\right]
\end{aligned}
$$

The generalized stresses and displacements (3.18) are presented graphically in Fig.2.
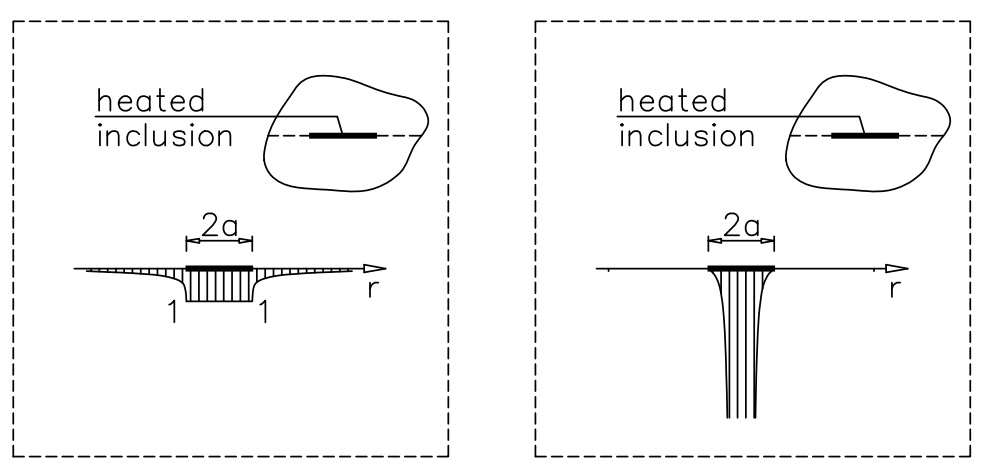

Fig.2. The generalized stresses and displacements on the plane $z=0$; are given by Eq.(3.19).

\section{The half - space problem}

We assume that the multifield material changes the temperature on the boundary which is given in the circular region $r \leq a$ as the constant cylindrical, revolution conical or revolution parabolic(see Fig.3).
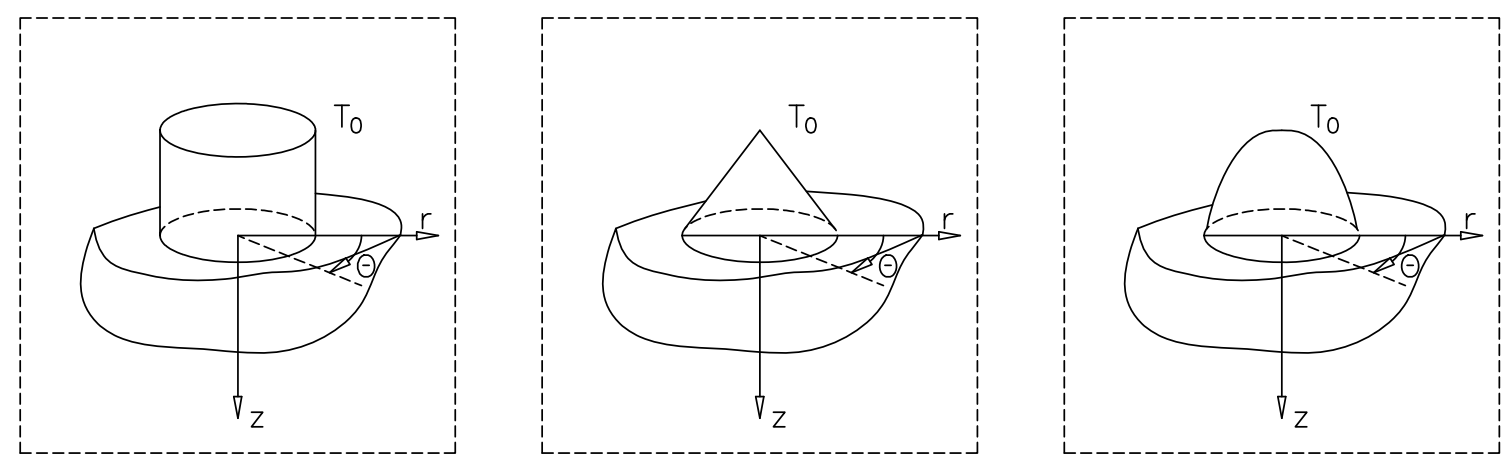

Fig.3. The half - space under temperature loading: a) cylindrical constant, b) revolution conical, c) revolution parabolic. 
The electric permittivity and magnetic permeability of the external medium (usually air) is much less that the multifield half - space region. For example $\varepsilon_{11} / \varepsilon_{\text {air }}=60 / 0,0885=680$ times lower for the PZT -4 commercial piezoelectric and $\mu_{11} / \mu_{\text {air }}=590 / 0,4 \pi=475$ times lower for a typical piezomagnetic. In consequence the electric displacement $D_{z}$ and magnetic induction $B_{z}$ must vanish on the boundary surface, which is also stress free.

Thus, we consider the following boundary conditions

$$
\sigma_{z r}=0, \quad \sigma_{z}+\sigma_{T}=0, \quad D_{z}+D_{T}=0, \quad B_{z}+B_{T}=0, \quad z=0, \quad r \geq 0
$$

where

$$
\left[\begin{array}{c}
\sigma_{T} \\
D_{T} \\
B_{T}
\end{array}\right]=T_{0}\left[\begin{array}{c}
\tilde{\beta} / \lambda_{0} \\
p \\
\gamma
\end{array}\right]\left[1-\left(1-\frac{2}{\pi} \arcsin \left(\frac{a}{r}\right) H\left(1-\frac{a}{r}\right)\right)\right]
$$

The quasi - harmonic functions needed for satisfying the boundary conditions are

$$
\phi_{i}\left(r, \lambda_{i} z\right)=\int_{0}^{\infty} B_{i}(\xi) e^{-\lambda_{i} \xi z} J_{0}(\xi r) d \xi
$$

where $J_{0}(\xi r)$ is the Bessel function and $\xi$ is a parameter of the Hankel transform.

The mixed boundary conditions on the plane $z=0$ give

$$
\begin{aligned}
& \sigma_{r z}(r, 0)=\sum_{i=1}^{4} \int_{0}^{\infty} \xi^{2} B_{i}(\xi) J_{l}(\xi r) d \xi=0, \quad r \geq 0, \\
& \begin{aligned}
\left\{\sigma_{z z}, D_{z}, B_{z}(r, 0)\right\} & =-\left[1-\left(1-\frac{2}{\pi} \arcsin \left(\frac{a}{r}\right) H\left(1-\frac{a}{r}\right)\right)\right]\left\{\tilde{\beta} \frac{1}{\lambda_{0}}, p, \gamma\right\} T_{0}= \\
& =\sum_{i=1}^{4}\left\{\frac{a_{5 i}}{\lambda_{i}}, a_{6 i} \lambda_{i}, a_{7 i} \lambda_{i}\right\} \int_{0}^{\infty} \xi^{2} B_{i}(\xi) J_{0}(\xi r) d \xi .
\end{aligned}
\end{aligned}
$$

To satisfy the zero condition at $z=0$ for $\sigma_{z z}, D_{z}$ and $B_{z}$ we obtain the additional displacements $u_{T}$, $\varphi_{T}$ and $\psi_{T}$. This thermal problem is obtained as given below.

Boundary conditions for generalized stresses yield

$$
\left[\begin{array}{c}
\xi B_{1}(\xi) \\
\xi B_{2}(\xi) \\
\xi B_{3}(\xi) \\
\xi B_{4}(\xi)
\end{array}\right]=-\frac{2}{\pi} T_{0} \frac{\sin \xi a}{\xi^{2}}\left[\begin{array}{cccc}
a_{51} & a_{52} & a_{53} & a_{54} \\
\frac{a_{51}}{\lambda_{1}} & \frac{a_{52}}{\lambda_{2}} & \frac{a_{53}}{\lambda_{3}} & \frac{a_{54}}{\lambda_{4}} \\
a_{61} \lambda_{1} & a_{62} \lambda_{2} & a_{63} \lambda_{3} & a_{64} \lambda_{4} \\
a_{71} \lambda_{1} & a_{72} \lambda_{2} & a_{73} \lambda_{3} & a_{74} \lambda_{4}
\end{array}\right]^{-1}\left[\begin{array}{c}
0 \\
\tilde{\beta} \frac{1}{\lambda_{0}} \\
p \\
\gamma
\end{array}\right],
$$


or

$$
\tilde{m}_{2} \xi B_{i}(\xi)=-\frac{2}{\pi} T_{0} \frac{\sin \xi a}{\xi^{2}} \Delta_{i}^{T}, \quad \Delta_{i}^{T}=\frac{1}{\lambda_{0}} \beta \tilde{d}_{i}+p \tilde{l}_{i}+\gamma \tilde{k}_{i}
$$

We have

$$
\begin{aligned}
& \sum a_{5 i} \tilde{d}_{i}=\sum a_{5 i} \tilde{l}_{i}=\sum a_{5 i} \tilde{k}_{i}=\sum a_{6 i} \lambda_{i} \tilde{d}_{i}=\sum a_{6 i} \lambda_{i} \tilde{k}_{i}=\sum a_{7 i} \lambda_{i} \tilde{d}_{i}=\sum a_{7 i} \lambda_{i} \tilde{l}_{i}=0, \\
& \tilde{m}_{2}=\sum \frac{a_{5 i}}{\lambda_{i}} \tilde{d}_{i}=\sum a_{6 i} \lambda_{i} \tilde{l}_{i}=\sum a_{7 i} \lambda_{i} \tilde{k}_{i}, \quad \sum=\sum_{i=1}^{4}, \quad \tilde{m}_{2}=\operatorname{det}(M)
\end{aligned}
$$

$M$ is the $4 \times 4$ matrix with elements $M_{j i}$ as in Eq.(4.5) (in this equation "-1" denotes the inverse matrix).

The $\tilde{d}_{i}, \tilde{l}_{i}$ and $\tilde{k}_{i}$ are the corresponding algebraic cofactors of matrix $M$ with elements $M_{j i}$ for $j=2,3$, and 4 , respectively (see equations A7).

The physical thermal fields in the multifield half - space are obtained as follows

$$
\begin{aligned}
& u_{r}^{T}(r, z)=-\frac{T_{0} r a_{10} \lambda_{0}}{\pi a_{00}}\left(\frac{\pi}{2}-\tan ^{-1} \xi_{0}+\frac{\xi_{0}}{1+\xi_{0}} \frac{1-\eta_{0}}{1+\eta_{0}}\right)+ \\
& +\frac{T_{0} r}{\pi} \sum_{i=1}^{4} a_{1 i} \lambda_{i} \tilde{\Delta}_{i}^{T}\left(\frac{\pi}{2}-\tan ^{-1} \xi_{i}+\frac{\xi_{i}}{1+\xi_{i}^{2}} \frac{1-\eta_{i}}{1+\eta_{i}}\right), \\
& {\left[\begin{array}{c}
u_{z}^{T}(r, z) \\
\varphi(r, z) \\
\psi(r, z)
\end{array}\right]=\frac{2 T_{0} a}{\pi a_{00}}\left[\begin{array}{c}
1 \\
-a_{30} \\
-a_{40}
\end{array}\right]\left[\frac{1}{2} \ln \frac{1+\eta_{0}}{1-\eta_{0}}-\eta_{0}\left(1-\xi_{0} \tan ^{-1} \frac{1}{\xi_{0}}\right)\right]+} \\
& -\frac{2 T_{0} a}{\pi} \sum_{i=1}^{4}\left[\begin{array}{c}
1 \\
-a_{3 i} \\
-a_{4 i}
\end{array}\right] \tilde{\Delta}_{i}^{T}\left[\frac{1}{2} \ln \frac{1+\eta_{i}}{1-\eta_{i}}-\eta_{i}\left(1-\xi_{i} \tan ^{-1} \frac{1}{\xi_{i}}\right)\right], \\
& {\left[\begin{array}{c}
\sigma_{z r}^{T}(r, z) \\
E_{r}^{T}(r, z) \\
H_{r}^{T}(r, z) \\
D_{r}^{T}(r, z) \\
B_{r}^{T}(r, z)
\end{array}\right]=\frac{2 T_{0}}{\pi a_{00}} \frac{a}{r}\left[\begin{array}{c}
a_{50}+\frac{\beta_{1}}{\lambda_{0}} a_{00} \\
a_{30} \\
a_{40} \\
a_{60} \lambda_{0}^{2} \\
a_{70} \lambda_{0}^{2}
\end{array}\right]\left(1-\eta_{0}\right)-\frac{2 T_{0}}{\pi} \frac{a}{r}\left[\begin{array}{c}
a_{5 i} \\
a_{3 i} \\
a_{4 i} \\
a_{6 i} \lambda_{i} \\
a_{70} \lambda_{i}
\end{array}\right] \widetilde{\Delta}_{i}^{T}\left(1-\eta_{i}\right),} \\
& {\left[\begin{array}{c}
\sigma_{z z}^{T}(r, z) \\
E_{z}^{T}(r, z) \\
H_{z}^{T}(r, z) \\
D_{z}^{T}(r, z) \\
B_{z}^{T}(r, z)
\end{array}\right]=\frac{2 T_{0}}{\pi a_{00}}\left[\begin{array}{c}
\left.a_{50}+\frac{\beta_{1}}{\lambda_{0}} a_{00}\right) \frac{1}{\lambda_{0}} \\
a_{30} \lambda_{0} \\
a_{40} \lambda_{0} \\
a_{60} \lambda_{0} \\
a_{70} \lambda_{0}
\end{array}\right]\left(\frac{\pi}{2}-\tan ^{-1} \xi_{0}\right)-\frac{2 T_{0}}{\pi} \sum_{i=1}^{4}\left[\begin{array}{c}
\frac{a_{5 i}}{\lambda_{i}} \\
a_{3 i} \lambda_{i} \\
a_{4 i} \lambda_{i} \\
a_{6 i} \lambda_{i} \\
a_{70} \lambda_{i}
\end{array}\right] \tilde{\Delta}_{i}^{T}\left(\frac{\pi}{2}-\tan ^{-1} \xi_{i}\right),}
\end{aligned}
$$




$$
\begin{aligned}
& {\left[\begin{array}{c}
\sigma_{r r}^{T}(r, z) \\
\sigma_{\theta \theta}^{T}(r, z)
\end{array}\right]=-\frac{2 T_{0}}{\pi a_{00}}\left(a_{50}+\frac{\beta_{1}}{\lambda_{0}} a_{00}\right)\left(\frac{\pi}{2}-\tan ^{-1} \xi_{0}\right)+} \\
& +\frac{2 T_{0}}{\pi} \sum_{i=1}^{4} a_{5 i} \lambda_{i} \tilde{\Delta}_{i}^{T}\left(\frac{\pi}{2}-\tan ^{-1} \xi_{i}\right)-\left(c_{11}-c_{12}\right)\left[\begin{array}{c}
\frac{u_{r}^{T}(r, z)}{r} \\
\frac{\partial u_{r}^{T}(r, z)}{\partial r}
\end{array}\right]
\end{aligned}
$$

where

$$
\tilde{\Delta}_{i}^{T}=\frac{\Delta_{i}^{T}}{\tilde{m}_{2}}+\frac{\beta \lambda_{0} d_{i}^{*}}{m_{2}^{*}}=\beta\left(\frac{\tilde{d}}{\tilde{m}_{2}}+\lambda_{0} \frac{d_{i}^{*}}{m_{2}^{*}}\right)+\frac{p \tilde{l}_{i}+\gamma \tilde{k}_{i}}{\tilde{m}_{2}}
$$

Note that on the boundary $z=0$ the coordinates $\xi_{i}$ and $\eta_{i}$ are independent of eigenvalues $\lambda_{i}$ and are

$$
\begin{aligned}
& \xi=0, \quad r \leq a, \quad a \xi=\sqrt{r^{2}-a^{2}}, \quad r \geq a, \\
& a \eta=\sqrt{a^{2}-r^{2}}, \quad r \leq a, \quad \eta=0, \quad r \geq a
\end{aligned}
$$

On the plane $z=0$ we obtain

$$
\begin{aligned}
& {\left[\begin{array}{c}
u_{z}^{T}(r, 0) \\
\varphi^{T}(r, 0) \\
\psi^{T}(r, 0)
\end{array}\right]=-\frac{2 T_{0} a}{\pi}\left[\begin{array}{c}
\alpha \\
\tilde{p} \\
\tilde{\gamma}
\end{array}\right]\left\{\begin{array}{l}
\ln \left(\frac{a}{r}+\sqrt{\frac{a^{2}}{r^{2}}-1}\right)-\sqrt{1-\frac{r^{2}}{a^{2}}}, \quad r \leq a \\
0 \quad r \geq a
\end{array}\right.} \\
& {\left[\begin{array}{c}
\alpha \\
\tilde{p} \\
\tilde{\gamma}
\end{array}\right]=\frac{1}{a_{00}}\left[\begin{array}{c}
1 \\
-a_{30} \\
-a_{40}
\end{array}\right]-\sum_{i=1}^{4}\left[\begin{array}{c}
1 \\
-a_{3 i} \\
-a_{4 i}
\end{array}\right],} \\
& {[\alpha]=10^{-6} 1 / \mathrm{K}, \quad[\tilde{p}]=10^{-6} \mathrm{~V} / \mathrm{mK}, \quad[\tilde{\gamma}]=10^{-4} \mathrm{~A} / \mathrm{mK},} \\
& \sigma_{z z}^{T}(r, 0)=T_{0}\left[1-\left(1-\frac{2}{\pi} \arcsin \frac{a}{r}\right) H\left(1-\frac{a}{r}\right)\right](\beta-\beta)=0 \\
& \sigma_{z r}^{T}(r, 0)=\frac{2}{\pi} T_{0} \frac{a-\sqrt{a^{2}-r^{2}}}{r}\left(\lambda_{0} \beta-\lambda_{0} \beta\right)=0, \\
& D_{z}^{T}(r, 0)=T_{0}\left[1-\left(1-\frac{2}{\pi} \arcsin \frac{a}{r}\right) H\left(1-\frac{a}{r}\right)\right]\left(p_{3}+p_{3}^{*}-p_{3}^{* *}-p\right)=0 \\
& B_{z}^{T}(r, 0)=T_{0}\left[1-\left(1-\frac{2}{\pi} \arcsin \frac{a}{r}\right) H\left(1-\frac{a}{r}\right)\right]\left(\gamma_{3}+\gamma_{3}^{*}-\gamma_{3}^{* *}-\gamma\right)=0
\end{aligned}
$$


The coefficients $\alpha, \tilde{p}$, and $\tilde{\gamma}$ may be called "the thermal coefficients of generalized compliances". The generalized displacements $u, \varphi$ and $\psi$ vanish on the boundary $z=0, r \geq a$ and as Fig.2 shows are regular except of the point $r=0$, where the solution has logarithmic singularity. All physical quantities satisfy the regularity conditions at infinity.

\section{Punch problem}

We assume that the cylindrical punch is flat ended, maintained at a constant electric and magnetic potential and temperature $T_{0}$ and loaded centrally by a concentrated force $P$ and by a concentrated electric charge $Q$ and total magnetic flux $B$.

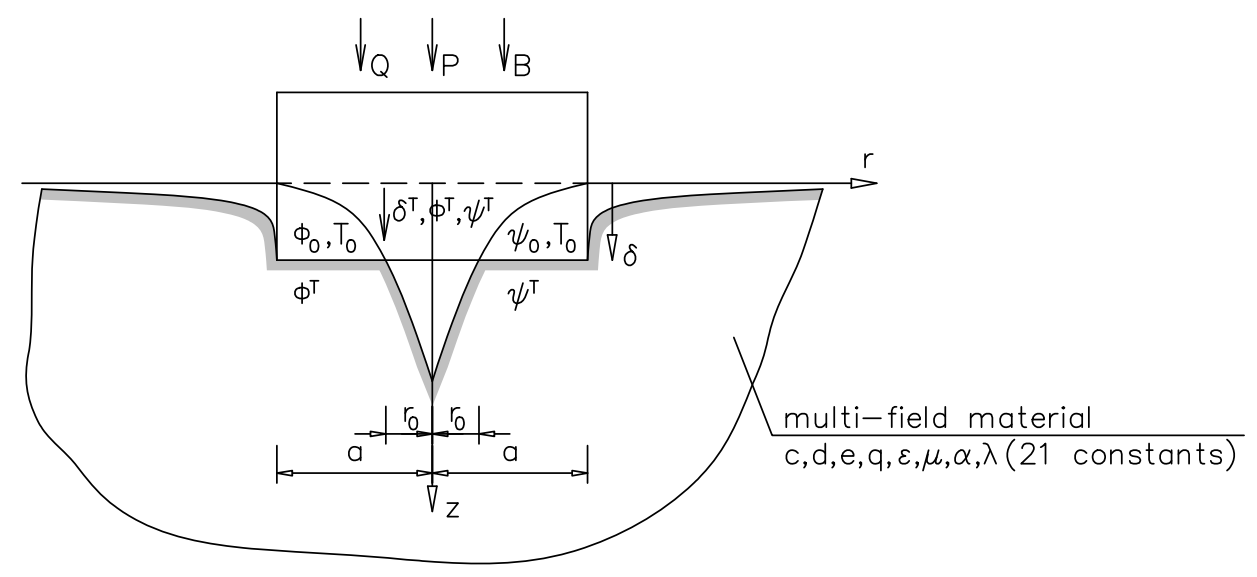

Fig.4. Punch on multifield half - space.

The contact region is annular

$$
r_{0} \leq r \leq a
$$

where $r_{0}$ is determined by the condition (see Fig.4)

$$
\delta=\delta^{T}\left(r_{0}\right)=\frac{2 T_{0} a}{\pi} \alpha\left[\ln \left(\frac{a}{r_{0}}+\sqrt{\frac{a^{2}}{r_{0}^{2}}-1}\right)-\sqrt{1-\frac{r_{0}^{2}}{a^{2}}}\right] .
$$

The inner circumference of the annulus will shrink with increasing load.

The boundary conditions are

$$
\begin{aligned}
& {\left[\begin{array}{c}
u_{z}(r, 0) \\
\phi(r, 0) \\
\psi(r, 0)
\end{array}\right]=\left[\begin{array}{c}
\delta \\
\phi_{0}+\phi^{T}(r) \\
\psi_{0}+\psi^{T}(r)
\end{array}\right], \quad r_{0} \leq r \leq a,} \\
& \sigma_{z z}(r, 0)=0, \quad r \geq 0,
\end{aligned}
$$




$$
\left[\begin{array}{c}
\sigma_{z z}(r, 0) \\
D_{z}(r, 0) \\
B_{z}(r, 0)
\end{array}\right]=\left[\begin{array}{l}
0 \\
0 \\
0
\end{array}\right], \quad r<r_{0}, \quad r>a
$$

Using the Hankel transform method the integral equations become

$$
\begin{aligned}
& {\left[\begin{array}{c}
u_{z}(r, 0) \\
\varphi(r, 0) \\
\psi(r, 0)
\end{array}\right]=\int_{0}^{\infty} \xi\left[\begin{array}{c}
-U(\xi) \\
\Phi(\xi) \\
\Psi(\xi)
\end{array}\right] J_{0}(r \xi) d \xi=\left[\begin{array}{c}
\delta \\
\varphi_{0}+\varphi^{T}(r) \\
\psi_{0}+\psi^{T}(r)
\end{array}\right], \quad r_{0} \leq r \leq a,} \\
& {\left[\begin{array}{c}
\sigma_{z z}(r, 0) \\
D_{z}(r, 0) \\
B_{z}(r, 0)
\end{array}\right]=[C] \int_{0}^{\infty} \xi\left[\begin{array}{c}
U(\xi) \\
\Phi(\xi) \\
\Psi(\xi)
\end{array}\right] J_{0}(r \xi) d \xi=0, \quad r_{0} \leq r \leq a}
\end{aligned}
$$

where $C$ is the indentation stiffness matrix defined as follows

$$
[C]=\frac{1}{m_{2}} \sum_{i=1}^{4}\left[\begin{array}{ccc}
a_{5 i} d_{i} / \lambda_{i} & a_{5 i} l_{i} / \lambda_{i} & a_{5 i} k_{i} / \lambda_{i} \\
a_{6 i} \lambda_{i} d_{i} & a_{6 i} \lambda_{i} l_{i} & a_{6 i} \lambda_{i} k_{i} \\
a_{7 i} \lambda_{i} d_{i} & a_{7 i} \lambda_{i} l_{i} & a_{7 i} \lambda_{i} k_{i}
\end{array}\right]=\frac{1}{m_{2}}\left[\begin{array}{ccc}
m & m_{6} & \tilde{m}_{6} \\
m_{5} & m_{7} & m_{8} \\
m_{9} & m_{10} & m_{11}
\end{array}\right],
$$

and where $d_{i}, l_{i}$ and $k_{i}$ are the corresponding algebraic cofactors of the multifield compliance matrix $M(4 \times 4)$ with elements $M_{j i},(i=1,2,3,4, j=2,3,4,5)$, for $j=2, j=3$ and $j=4$, respectively

$$
[M]=\left[\begin{array}{cccc}
1 & 1 & 1 & 1 \\
a_{31} & a_{32} & a_{33} & a_{34} \\
a_{41} & a_{42} & a_{43} & a_{44} \\
a_{51} & a_{52} & a_{53} & a_{54}
\end{array}\right]
$$

Then, if $m_{2}=\operatorname{det}(M)$, the material parameters $d_{i}, l_{i}$ and $k_{i}$ are

$$
\begin{aligned}
& M\left(d_{1}, d_{2}, d_{3}, d_{4}\right)^{T}=\left(m_{2}, 0,0,0\right)^{T}, \\
& M\left(l_{1}, l_{2}, l_{3}, l_{4}\right)^{T}=\left(0, m_{2}, 0,0\right)^{T}, \\
& M\left(k_{1}, k_{2}, k_{3}, k_{4}\right)^{T}=\left(0,0, m_{2}, 0\right)^{T}
\end{aligned}
$$

here $T$ denotes the transpose of a matrix. The condition (5.4) is satisfied identically. We have

$$
\begin{aligned}
& m_{2}=\sum d_{i}=\sum a_{3 i} l_{i}=\sum a_{4 i} k_{i}, \\
& \sum l_{i}=\sum k_{i}=\sum a_{3 i} d_{i}=\sum a_{3 i} k_{i}=\sum a_{4 i} d_{i}=\sum a_{4 i} l_{i}=\sum a_{5 i} d_{i}=\sum a_{5 i} l_{i}=\sum a_{5 i} k_{i}=0
\end{aligned}
$$


where the sum is from 1 to 4 . The coefficients $d_{i}, l_{i}$ and $k_{i}$ are given in the Appendix A by Eqs (A6).

The integral equations become

$$
\begin{aligned}
& \int_{0}^{\infty} \xi\left[\begin{array}{c}
-U(\xi) \\
\Phi(\xi) \\
\Psi(\xi)
\end{array}\right] J_{0}(r \xi) d \xi=\left[\begin{array}{c}
\delta \\
\varphi_{0}+\varphi^{T}(r) \\
\psi_{0}+\psi^{T}(r)
\end{array}\right], \quad r_{0} \leq r \leq a \\
& {[C] \int_{0}^{\infty} \xi^{2}\left[\begin{array}{c}
U(\xi) \\
\Phi(\xi) \\
\Psi(\xi)
\end{array}\right] J_{0}(r \xi) d \xi=0, \quad r<r_{0}, \quad r>a}
\end{aligned}
$$

Changing the variable $r \in\left\langle r_{0}, a\right\rangle$ on $\alpha \in\langle 0, \pi\rangle$ by equation

$$
2 r_{c} b \cos \alpha=r_{c}^{2}+b^{2}-r^{2}, \quad a=r_{c}+b, \quad r_{0}=r_{c}-b,
$$

and assuming that $\xi U(\xi), \xi \varphi(\xi), \xi \psi(\xi)$ can be presented by integral as follows

$$
\begin{aligned}
& \xi\left[\begin{array}{c}
U(\xi) \\
\varphi(\xi) \\
\psi(\xi)
\end{array}\right]=\int_{0}^{\infty}\left[\begin{array}{l}
F_{1}(\beta) \\
F_{2}(\beta) \\
F_{3}(\beta)
\end{array}\right] J_{0}(\xi R) d \beta, \\
& R^{2}=r_{c}^{2}+b^{2}-2 r_{c} b \cos \beta,
\end{aligned}
$$

we obtain

$$
\left[\begin{array}{c}
\sigma_{z z}(r, 0) \\
D_{z}(r, 0) \\
B_{z}(r, 0)
\end{array}\right]=-[C] \int_{0}^{\infty}\left[\begin{array}{c}
F_{1}(\beta) \\
F_{2}(\beta) \\
F_{3}(\beta)
\end{array}\right] \frac{\delta(R-r)}{\sqrt{R r}} d \beta .
$$

Here $F_{1}(\beta), F_{2}(\beta)$ and $F_{3}(\beta)$ are arbitrary continuous functions, $\delta(R-r)$ is Dirac's delta function and the following formula is used

$$
\int_{0}^{\infty} \xi J_{0}(\xi R) J_{0}(\xi r) d \xi=\frac{\delta(R-r)}{\sqrt{R r}}
$$

Then Eqs (5.13) are identically satisfied. Introducing the series representations

$$
\left[\begin{array}{l}
F_{1}(\beta) \\
F_{2}(\beta) \\
F_{3}(\beta)
\end{array}\right]=\sum_{n=0}^{\infty}\left[\begin{array}{l}
a_{n} \\
b_{n} \\
c_{n}
\end{array}\right] \cos (n \beta),
$$


we obtain from Eqs (5.16)

$$
\left[\begin{array}{c}
\sigma_{z z}(r, 0) \\
D_{z}(r, 0) \\
B_{z}(r, 0)
\end{array}\right]=-[C] \frac{1}{r_{c} b \sin \alpha} \sum_{n=0}^{\infty}\left[\begin{array}{l}
a_{n} \\
b_{n} \\
c_{n}
\end{array}\right] \cos (n \beta) .
$$

Substituting Eqs (5.18) and (5.15) into the boundary conditions (5.12), we obtain

$$
\sum_{n=0}^{\infty}\left[\begin{array}{l}
a_{n} \\
b_{n} \\
c_{n}
\end{array}\right] \int_{0}^{\infty} J_{0}(\xi r) d \xi \int_{0}^{\pi} J_{0}(\xi R) \cos (n \beta) d \beta=\left[\begin{array}{c}
\delta \\
-\varphi_{0}-\varphi^{T}(r) \\
-\psi_{0}-\psi^{T}(r)
\end{array}\right] .
$$

Using the formula (Gradsztejn and Ryzhik, 1965)

$$
\int_{0}^{\pi} J_{0}(\xi R) \cos (n \beta) d \beta=\pi J_{n}\left(\xi r_{c}\right) J_{n}(\xi b)=\pi Z_{n}\left(\xi r_{c}, \xi b\right) .
$$

Then Eq.(5.20) becomes

$$
\sum_{n=0}^{\infty}\left[\begin{array}{l}
a_{n} \\
b_{n} \\
c_{n}
\end{array}\right] \int_{0}^{\infty} J_{0}(\xi r) Z_{n}\left(\xi r_{c}, \xi b\right) d \xi=\frac{1}{\pi}\left[\begin{array}{c}
\delta \\
-\varphi_{0}-\varphi^{T}(r) \\
-\psi_{0}-\psi^{T}(r)
\end{array}\right] .
$$

Introducing the new coefficients $d_{n}$ and $e_{n}$ instead of $b_{n}$ and $f_{n}$ and $g_{n}$ instead of $c_{n}$ as follows

$$
\begin{aligned}
& b_{n}=-\frac{a}{\pi} \varphi_{0} d_{n}-\frac{2 T_{0} a^{2}}{\pi^{2}} \tilde{p} e_{n}, \\
& c_{n}=-\frac{a}{\pi} \psi_{0} f_{n}-\frac{2 T_{0} a^{2}}{\pi^{2}} \tilde{\gamma} g_{n},
\end{aligned}
$$

Eq.(5.22) are converted to five algebraic equations with respect to $a_{n}, d_{n}, e_{n}, f_{n}$ and $g_{n}$

$$
\sum_{n=0}^{\infty}\left[\begin{array}{c}
a_{n} \\
d_{n} \\
e_{n} \\
f_{n} \\
g_{n}
\end{array}\right] \int_{0}^{\infty} J_{0}(\xi r) Z_{n}\left(\xi r_{c}, \xi b\right) d \xi=\left[\begin{array}{c}
1 \\
1 \\
h(r) \\
1 \\
h(r)
\end{array}\right], \quad r_{0} \leq r \leq a
$$

where 


$$
h(r)=\ln \left(\frac{a}{r}+\sqrt{\frac{a^{2}}{r^{2}}-1}\right)-\sqrt{1-\frac{r^{2}}{a^{2}}}, \quad r \leq a .
$$

We conclude that $a_{n}=d_{n}=f_{n}$ and $e_{n}=g_{n}$. By using Neumann's formula (Gradsztejn and Ryzhik, 1965)

$$
J_{0}(\xi r)=Z_{0}\left(\xi r_{c}, \xi b\right)+2 \sum_{m=1}^{\infty} Z_{m}\left(\xi r_{c}, \xi b\right) \cos (m \alpha), \quad 0 \leq \alpha \leq \pi
$$

and the Fourier expansion for the function $h(r)$

$$
\begin{aligned}
& h(r)=h_{0}+2 \sum h_{m} \cos (m \alpha), \\
& h_{m}=\frac{1}{\pi} \int_{0}^{\pi} h(r) \cos (m \alpha) d \alpha,
\end{aligned}
$$

Eqs.(5.24) are converted to two simultaneous algebraic equations

$$
\begin{aligned}
& \sum_{n=0}^{\infty} a_{n} A_{m n}=\delta_{0 m}, \\
& \sum_{n=0}^{\infty} e_{n} A_{m n}=h_{m}, \quad m=0,1,2, \ldots
\end{aligned}
$$

with the matrix

$$
\begin{aligned}
& A_{m n}=\int_{0}^{\infty} J_{m}\left(\xi s_{1}\right) J_{m}\left(\xi s_{2}\right) J_{n}\left(\xi s_{1}\right) J_{n}\left(\xi s_{2}\right) d \xi, \\
& s_{1,2}=\frac{1}{2}(1 \pm s), \quad s=\frac{r_{0}}{a},
\end{aligned}
$$

and where $\delta_{0 m}$ is Kronecker's delta.

From the condition that $\sigma_{z z}(r, 0)$ must be finite at $r=r_{0}(\alpha=0)$ we conclude that the infinite series in Eqs (5.19) must be zero for $\alpha \rightarrow O\left(r \rightarrow r_{0}+0\right)$. This gives the condition

$$
\frac{\sum_{n=0}^{\infty} a_{n}}{\sum_{n=0}^{\infty} e_{n}}=\frac{\frac{2 T_{0} a}{\pi \delta}\left(\tilde{p} \frac{m_{6}}{m}+\widetilde{\gamma} \frac{\tilde{m}_{6}}{m}\right)}{1-\frac{m_{6}}{m} \frac{\phi_{0}}{\delta}-\frac{\tilde{m}_{6}}{m} \frac{\psi_{0}}{\delta}} .
$$


The equation gives the relation between the depth of penetration of the punch $\delta$, the boundary electric and magnetic potentials $\varphi_{0}$ and $\psi_{0}$ and the radius of the contact region $r_{0}$, since the sets $a_{n}$ and $e_{n}$ depend on $r_{0}$. Of course, temperature $T_{0}$ appears in this equation.

Finally, we obtain

a) contact stress

$$
\sigma_{z z}(r, 0)=-\frac{a \delta m}{\pi r_{c} b m_{2}} \frac{1}{\sin \alpha} \sum_{n=0}^{\infty}\left[a_{n}\left(1-\frac{\varphi_{0}}{\delta} \frac{m_{6}}{m}-\frac{\psi_{0}}{\delta} \frac{\tilde{m}_{6}}{m}\right)-e_{n} \frac{2 T_{0} a}{\pi \delta}\left(\tilde{p} \frac{m_{6}}{m}+\tilde{\gamma} \frac{\tilde{m}_{6}}{m}\right)\right],
$$

b) electric displacement

$$
D_{z}(r, 0)=-\frac{a \delta m_{5}}{\pi r_{c} b m_{2}} \frac{1}{\sin \alpha} \sum_{n=0}^{\infty}\left[a_{n}\left(1-\frac{\varphi_{0}}{\delta} \frac{m_{7}}{m_{5}}-\frac{\psi_{0}}{\delta} \frac{m_{8}}{m_{5}}\right)-e_{n} \frac{2 T_{0} a}{\pi \delta}\left(\tilde{p} \frac{m_{7}}{m_{5}}+\tilde{\gamma} \frac{m_{8}}{m_{5}}\right)\right],
$$

c) magnetic induction

$$
B_{z}(r, 0)=-\frac{a \delta m_{9}}{\pi r_{c} b m_{2}} \frac{1}{\sin \alpha} \sum_{n=0}^{\infty}\left[a_{n}\left(1-\frac{\phi_{0}}{\delta} \frac{m_{10}}{m_{9}}-\frac{\psi_{0}}{\delta} \frac{m_{11}}{m_{9}}\right)-e_{n} \frac{2 T_{0} a}{\pi \delta}\left(\tilde{p} \frac{m_{10}}{m_{9}}+\widetilde{\gamma} \frac{m_{11}}{m_{9}}\right)\right],
$$

d) displacement on the boundary $z=0$

$$
u_{z}(r, 0)=\delta \sum_{n=0}^{\infty} a_{n} I_{0}^{n}\left(\frac{r}{a}\right)
$$

e) electric potential on the boundary $z=0$

$$
\varphi(r, 0)=-\varphi_{0} \sum_{n=0}^{\infty} a_{n} I_{0}^{n}\left(\frac{r}{a}\right)
$$

f) magnetic potential on the boundary $z=0$

$$
\psi(r, 0)=-\psi_{0} \sum_{n=0}^{\infty} a_{n} I_{0}^{n}\left(\frac{r}{a}\right)
$$

where the integrals

$$
I_{0}^{n}\left(\frac{r}{a}\right)=\int_{0}^{\infty} J_{0}\left(\xi \frac{r}{a}\right) J_{n}\left(\xi s_{1}\right) J_{n}\left(\xi s_{2}\right) d \xi
$$

are presented analytically, as well as the matrix $A_{m n}$ in Eq.(5.28), by Rogowski (2006).

It can be shown that the gradient of $u_{z}(r, 0)$ is bounded for $r \rightarrow r_{0}+0$ and unbounded for $r \rightarrow r_{0}-0$ and for $r \rightarrow a+0$ tending, to plus or minus infinity, respectively. If in the contact region 
potentials $\varphi_{0}$ and $\psi_{0}$ appear the gradient of $u_{z}(r, 0)$ tends to minus infinity at the left neighborhood of the contact circle $r=r_{0}$. This phenomenon occurs, among others, in adhesive contact problem.

The equilibrium of the punch yields

$$
P=2 \pi a \delta \frac{m}{m_{2}}\left[a_{0}\left(1-\frac{\varphi_{0}}{\delta} \frac{m_{6}}{m}-\frac{\psi_{0}}{\delta} \frac{\tilde{m}_{6}}{m}\right)-e_{0} \frac{2 T_{0} a}{\pi \delta}\left(\tilde{p} \frac{m_{6}}{m}+\tilde{\gamma} \frac{\tilde{m}_{6}}{m}\right)\right] .
$$

The coefficients $h_{m}$ are calculated from the formulae

$$
\begin{aligned}
& h_{m}=\frac{r_{c} b}{a^{2}}\left(2 \delta_{0 m}+\delta_{1 m}\right) \text { for } h(r)=1-\frac{r^{2}}{a^{2}}, \\
& h_{m}(0)=\frac{1}{4}\left(2 \delta_{0 m}+\delta_{1 m}\right) \text { for } r_{0}=0, \\
& h_{m}=\frac{1}{\pi a} \int_{0}^{a}\left(a-\sqrt{a^{2}+b^{2}-2 r_{c} b \cos \alpha}\right) \cos (m \alpha) d \alpha \text { for } h(r)=1-\frac{r}{a} .
\end{aligned}
$$

The solution of the infinite systems of algebraic Eqs (5.28) for $r_{0}=0$ is

$$
\begin{aligned}
& a_{n}=-\frac{4}{\pi\left(1+\delta_{n 0}\right)} \frac{1}{4 n^{2}-1}, \\
& e_{n}=-\frac{4}{\pi\left(1+\delta_{n 0}\right)}\left(\frac{1}{4 n^{2}-1}-\frac{6}{4 n^{2}-9}\right)
\end{aligned}
$$

where $\delta_{n 0}$ is Kronecker's delta.

Equations (5.38) and (5.40) yield

$$
P=4 \delta a \frac{m}{m_{2}}\left[1-\frac{\varphi_{0}}{\delta} \frac{m_{6}}{m}-\frac{\psi_{0}}{\delta} \frac{\tilde{m}_{6}}{m}-\frac{2}{3} \frac{T_{0} a}{\delta}\left(\tilde{p} \frac{m_{6}}{m}+\tilde{\gamma} \frac{\tilde{m}_{6}}{m}\right)\right],
$$

which is the solution of the problem ("theoretically") of full contact.

Note that the solution $\left(a_{n}, e_{n}\right)$ depends on the ratio of contact radii $r_{0} / a$ and the inner radius $r_{0}$ is unknown. Notice that for an annular contact region the solution $\left(a_{n}, e_{n}\right)$ of the simultaneous algebraic equations satisfies the inequalities

$$
\left|a_{n}, e_{n}(s \rightarrow 1)\right| \leq\left|a_{n}, e_{n}(s)\right| \leq\left|a_{n}, e_{n}(0)\right|, \quad s=\frac{r_{0}}{a} .
$$

The piezoelectric response amplitude $m_{6} / m$ and piezomagnetic response amplitude $\tilde{m}_{6} / m$ for real materials are negative. This proves that increasing the applied electric and / or magnetic potentials will 
always reduce the indentation depth $\delta$. Similarly, if $T_{0} \tilde{p}$ and / or $T_{0} \tilde{\gamma}$ are positive the indentation depth $\delta$ is smaller than that for isothermal problem. For negative $T_{0} \tilde{p}$ and / or $T_{0} \tilde{\gamma}$ the thermal generalized displacement $\varphi^{T}$ and $\psi^{T}$ change the sign and $\delta$ increases.

The piezoelectric and piezomagnetic response amplitudes are defined as follows

$$
\begin{aligned}
& \operatorname{Re} R=\left(\frac{\partial \delta}{\partial \varphi_{0}}\right)_{\substack{P=\text { const } \\
\psi_{0}=\text { const }}}=\frac{m_{6}}{m}\left[\frac{m}{V}\right], \\
& \operatorname{PmR}=\left(\frac{\partial \delta}{\partial \psi_{0}}\right)_{\substack{P=\text { const } \\
\varphi_{0}=\text { const }}}=\frac{\tilde{m}_{6}}{m}\left[\frac{m}{A}\right] .
\end{aligned}
$$

For $\mathrm{BaTiO}_{3}-\mathrm{CoFe}_{2} \mathrm{O}_{4}$ commercial composite we have

$$
\mathrm{PeR}=-2.14 \times 10^{-9} \mathrm{~m} / \mathrm{V}, \quad \mathrm{PmR}=-7.7 \times 10^{-9} \mathrm{~m} / \mathrm{A} .
$$

The indentation elastic stiffness coefficient

$$
\frac{P}{4 a \delta}=\frac{m}{m_{2}}
$$

assumes the values of $62.5 \mathrm{GPa}$ for the multifield composite $\mathrm{BaTiO}_{3}-\mathrm{CoFe}_{2} \mathrm{O}_{4}$ and $70.4 \mathrm{GPa}$ for a pure elastic transversely isotropic material with parameters $c_{i j}$ only for this composite.

The indentation piezoelectric coefficient is

$$
\frac{Q}{4 a \delta}=\frac{m_{5}}{m_{2}}=14,3 \frac{C}{m^{2}}
$$

The indentation piezomagnetic coefficient is

$$
\frac{B}{4 a \delta}=\frac{m_{9}}{m_{2}}=7.0 \times 10^{3} \frac{\mathrm{N}}{\mathrm{Am}},
$$

for this multifield material.

The total concentrated electric charge $Q$ and total magnetic flux $B$ in the contact region are obtained by integrating $D_{z}$ and $B_{z}$ over the circle of contact. We obtain

$$
\begin{aligned}
& Q=2 \pi a \delta \frac{m_{5}}{m_{2}}\left[a_{0}\left(1-\frac{\varphi_{0}}{\delta} \frac{m_{7}}{m_{5}}-\frac{\psi_{0}}{\delta} \frac{m_{8}}{m_{5}}\right)-e_{0} \frac{2 T_{0} a}{\pi \delta}\left(\tilde{p} \frac{m_{7}}{m_{5}}+\tilde{\gamma} \frac{m_{8}}{m_{5}}\right)\right], \\
& B=2 \pi a \delta \frac{m_{9}}{m_{2}}\left[a_{0}\left(1-\frac{\varphi_{0}}{\delta} \frac{m_{10}}{m_{9}}-\frac{\psi_{0}}{\delta} \frac{m_{11}}{m_{9}}\right)-e_{0} \frac{2 T_{0} a}{\pi \delta}\left(\tilde{p} \frac{m_{10}}{m_{9}}+\tilde{\gamma} \frac{m_{11}}{m_{9}}\right)\right] .
\end{aligned}
$$


The coupling terms $m_{6}, \tilde{m}_{6}, m_{8}$ (or $m_{5}, m_{9}, m_{10}$ ), which are the elements of the matrix on the right hand side of Eq.(5.8) are non - zero for multifield materials. This suggests that even in the absence of an applied electric and / or magnetic potentials, an electric and magnetic charge could be accumulated on the surface due to the applied mechanical load or displacement.

Similarly, an applied electric potential and / or magnetic potential could cause mechanical pressure at the contact region. Solving Eqs (5.38) and (5.47) with respect to $\delta, \varphi_{0}$ and $\psi_{0}$, we obtain the corresponding generalized compliance relations.

Defining the stress, electric displacement and magnetic induction intensity factors as follows

$$
\left[K_{\sigma}, K_{D}, K_{B}\right]^{T}=\lim _{r \rightarrow a^{-}} \sqrt{2(a-r)}\left[\sigma_{z}(r, 0), D_{z}(r, 0), B_{z}(r, 0)\right]^{T}
$$

we obtain

$$
\left[K_{\sigma}, K_{D}, K_{B}\right]^{T}=\frac{1}{2 \pi a \sqrt{a}}[P, Q, B]
$$

\section{Single phase materials and multifield composite materials}

Use the notation

$$
e_{1}=\left(e_{31}+e_{15}\right) \lambda_{i}^{2}, \quad e_{2}=e_{31}+e_{33} \lambda_{i}^{2}, \quad c=c_{11}+c_{13} \lambda_{i}^{2}, \quad \varepsilon_{1}=\varepsilon_{11}-\varepsilon_{33} \lambda_{i}^{2},
$$

and define the matrix and its inverse

$$
C_{E}=\left[\begin{array}{ccc}
e_{1} & \varepsilon_{1} & 0 \\
0 & 0 & -\infty \\
c & e_{2} & 0
\end{array}\right], \quad C_{E}^{-1}=\frac{1}{e_{1} e_{2}-c \varepsilon_{1}}\left[\begin{array}{ccc}
e_{2} & 0 & -\varepsilon_{1} \\
-c & 0 & e_{1} \\
0 & 0 & 0
\end{array}\right] .
$$

Of course, $C_{E} C_{E}^{-1}$ is the square unit matrix.

Similarly,

$$
\begin{aligned}
& q_{1}=\left(q_{31}+q_{15}\right) \lambda_{i}^{2}, \quad q_{2}=q_{31}+q_{33} \lambda_{i}^{2}, \quad c=c_{11}+c_{13} \lambda_{i}^{2}, \quad \mu_{1}=\mu_{11}-\mu_{33} \lambda_{i}^{2}, \\
& C_{H}=\left[\begin{array}{ccc}
0 & -\infty & 0 \\
q_{1} & 0 & \mu_{1} \\
c & 0 & q_{2}
\end{array}\right], \quad C_{H}^{-1}=\frac{1}{q_{1} q_{2}-c \mu_{1}}\left[\begin{array}{ccc}
0 & q_{2} & -\mu_{1} \\
0 & 0 & 0 \\
0 & -c & q_{1}
\end{array}\right] .
\end{aligned}
$$

Thus, we obtain

$$
\left\{\begin{array}{l}
a_{1 i} \\
a_{3 i}
\end{array}\right\}^{E}=\frac{1}{e_{1} e_{2}-c \varepsilon_{1}}\left[\begin{array}{cc}
e_{2} & -\varepsilon_{1} \\
-c & e_{1}
\end{array}\right]\left\{\begin{array}{c}
e_{33} \lambda_{i}^{2}-e_{15}+p_{3} a_{00} \lambda_{i} \delta_{i 0} \\
c_{33} \lambda_{i}^{2}+c_{13}-\left(\beta_{3}+\beta_{1} \lambda_{i}^{-2}\right) a_{00} \lambda_{i} \delta_{i 0}
\end{array}\right\},
$$




$$
\left\{\begin{array}{l}
a_{1 i} \\
a_{4 i}
\end{array}\right\}^{H}=\frac{1}{q_{1} q_{2}-c \mu_{1}}\left[\begin{array}{cc}
q_{2} & -\mu_{1} \\
-c & q_{1}
\end{array}\right]\left\{\begin{array}{c}
q_{33} \lambda_{i}^{2}-q_{15}+\gamma_{3} a_{00} \lambda_{i} \delta_{i 0} \\
c_{33} \lambda_{i}^{2}+c_{13}-\left(\beta_{3}+\beta_{1} \lambda_{i}^{-2}\right) a_{00} \lambda_{i} \delta_{i 0}
\end{array}\right\},
$$

respectively, for piezoelectric and piezomagnetic thermoelastic materials.

Note that for a piezoelectric material $a_{4 i} \equiv 0$, but $a_{3 i}$ define the coefficients $a_{7 i}$, that is also the magnetic induction $B_{z}$, by the electromagnetic constant $d_{11}$. Similarly, for the piezomagnetic material where $a_{3 i} \equiv 0, a_{4 i}$ define $a_{6 i}$, that is also $D_{z}$, as a consequence of the electromagnetic effect (see Eqs $(2.7))$.

For a composite two - phase $(\mathrm{E}+\mathrm{H})$ multifield material the compliance matrix may be defined as

$$
C_{E H}^{-1}=\frac{1}{2}\left(C_{E}^{-1}+C_{H}^{-1}\right)
$$

The constitutive stiffness matrix for the composite will be the inversion of this compliance matrix. The solution presented here may be used for composite materials made of multifield materials.

\section{Conclusions}

The potential harmonic theory method has been generalized in this paper to analyze the thermal Green's functions for a multifield material. Green's functions are used to analyze the contact problem of a heated and conducting punch indenting a multifield half - space. The boundary value problem is converted to triple integral equations, which are reduced to simultaneous two infinite systems of algebraic equations. In the limiting case of full contact, which theoretically may occur, the closed form of solution is obtained. The expressions for displacements, stresses, electric and magnetic potentials and electric displacement, and magnetic fluxes are presented in terms of infinite series. Some important relationships between the applied or accompanied loads and indentation depth, constant electric potential and magnetic potential are established. It is worth mentioning here again that the general solution shall take another form for equal eigenvalues cases. However, one can also derive the corresponding results of equal eigenvalues directly from the ones of distinct eigenvalues, by utilizing the well known l'Hospital rule.

In the light of the analytical analysis the following conclusions can be formulated.

1. Increasing the applied electric and / or magnetic potentials will always reduce the indentation depth of the punch.

2. The indentation electric stiffness for the representative multifield material $\mathrm{BaTiO}_{2}-\mathrm{CoFe}_{2} \mathrm{O}_{3}$ is smaller than that for a pure elastic transversely isotropic material.

3. The hypothesis of the constant electric and magnetic potential in the contact region is equivalent to a centrally applied concentrated charge $Q$ and magnetic induction $B$, which can be obtained by integrating electric displacement and / or magnetic flux over the annular contact region.

4. It can be seen from the obtained formulae that the complete solution can be separated into three parts: the first part corresponds to the normal displacement $\delta$, the second to the electric potential $\phi_{0}$ and the third to the magnetic potential $\psi_{0}$.

5. If the contact region electric and magnetic potentials occur (conducting punch), then the phenomenon similar to adhesive contact occurs in the left neighborhood of the inner radius of the contact region. 


\section{Nomenclature}

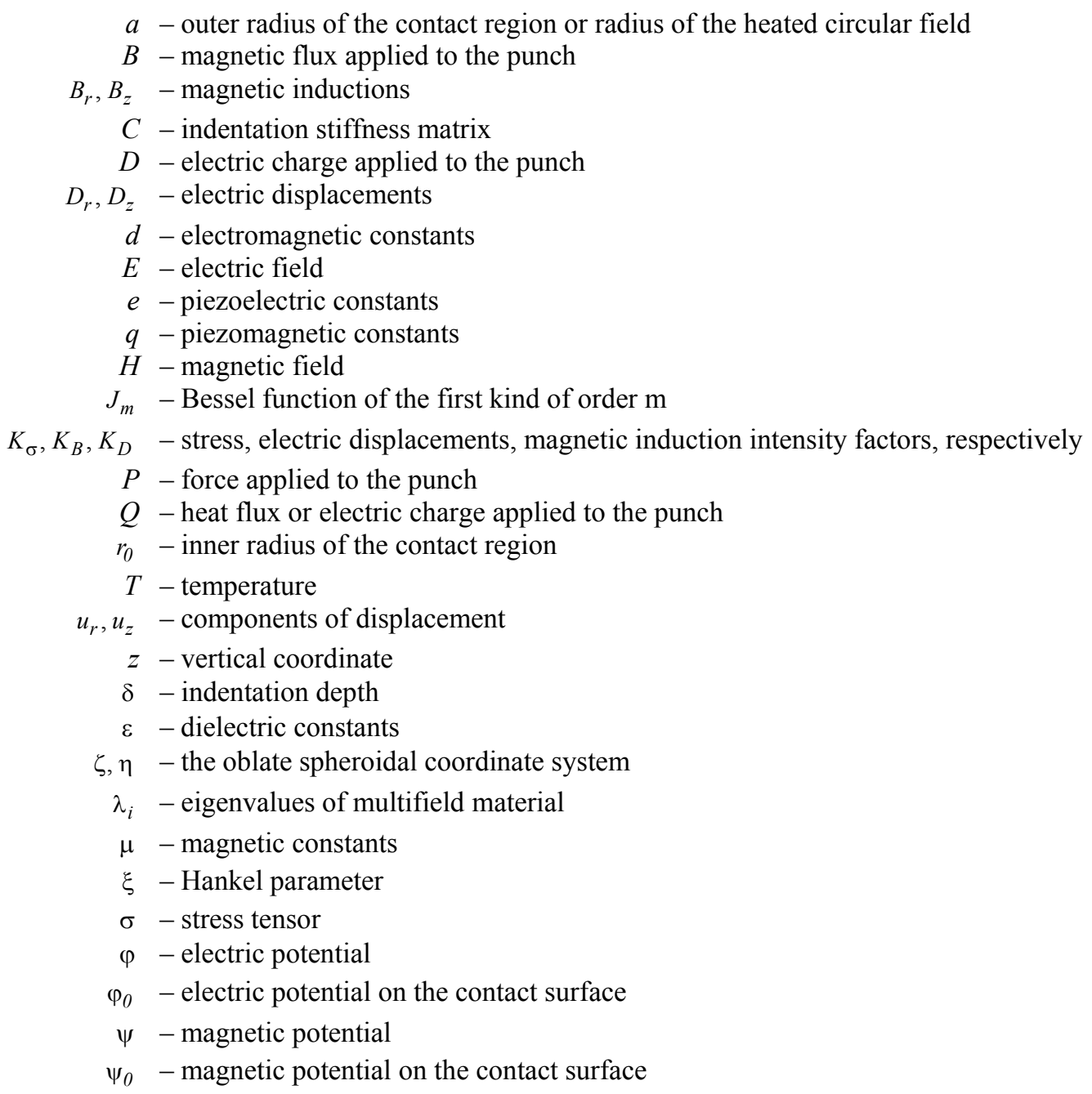

Other symbols are defined in the text of the paper.

\section{References}

Chen W., Pan E., Wang H. and Zhang C. (2010): Theory of indentation on multiferroic composite materials. - Journal of the Mechanics and Physics of Solids, vol.58, pp.1524-1551.

Gladwell G.M.L. (1980): Contact Problems in the Classical Theory of Elasticity. - Amsterdam: Sijthoff and Noorthoff.

Gradsztejn I.S. and Ryzhik I.M. (1965): Tables of Integrals Series and Products. - New York: Academic Press.

Hills D.A., Nowell D. and Sackfield A. (1993): Mechanics of Elastic Contacts. - Butterworth-Heinemann.

Johnson K.L. (1985): Contact Mechanics. - UK: Cambridge University Press.

Kalinin S.V., Karapetian E. and Kachanov M. (2004): Nanoelectromechanics of piezoresponse force microscopy. Physical Review B 70 184101(24).

Raous M., Jean M. and Moreau J.J. (1995): Contact Mechanics. - New York: Plenum Press.

Rogowski B. (2006): Contact Problems for Elastic Anisotropic Media. - A series of Monographs, Technical University of Lodz. 
Rogowski B. (2006): Inclusion Problems for Elastic Anisotropic Media. - A series of Monographs, Technical University of Lodz.

Rogowski B. (2012): A concave indenter on a piezo - electro - magneto - elastic substrate or a layer elastically supported. - The Journal of Strain Analysis for Engineering Design 47.6, pp.362-378.

Rogowski B. (2014): Contact problems of smart MEEMs. - Lambert Academic Publishing, Saarbrücken Deutschland, Germany.

Rogowski B. and Kaliński W. (2012): Indentation of piezoelectromagnetoelastic half - space by a truncated conical punch. - International Journal of Engineering Science, vol.60, pp.77-93.

\section{From author}

The Curie brothers discovered the piezoelectric effect in 1880 (Cady W. G., Piezoelectricity, New York, McGraw - Hill, 1964). Today, over a 100 piezoelectric materials or composites are known (Pohanka R. C., Smith P. L., Recent advances in piezoelectric ceramics, In: Levinson M. L., editor, Electronic ceramics, New York: Marcel Dekker, 1988).

The electro - mechanical, magneto - mechanical, electro - magnetic coupling of those materials, which I name "multifield materials", has an immense technological potential in designing "smart" materials and structures ranging from huge aerospace structures to miniaturized medical devices and miniatural medical apparatures. Multifield materials have been widely used for applications such as sensors, filters, ultrasonic generators and actuators, magnetic field probes, acoustic and ultrasonic devices, hydrophones. Also these materials have been employed as integrated active structural elements. These structures are capable of monitoring and adapting to their environment providing a "smart", response to the external conditions. Interested readers may refer to a state of the art survey by Rao S. S., Sunar M., Piezoelectricity and its use in disturbance sensing and control of flexible structures: a survey, Appl. Mech. Rev., 1994, 47, 113 - 123. One of the practical examples of a piezoelectric device is a piezoelectric accelerometer for triggering the onset of an airbag in tens of thousandths of a second during the accident. The advanced micro - electro - mechanical systems use piezoelectric materials in the latest technologies of smart / intelligent designs featuring miniaturization.

The physical law for piezoelectric materials has been explored by Nowacki $W$., in $1^{\circ}$ Some general theorems in thermo - piezoelectricity, J. Therm., Stresses, 1978, 1, 171 - 182, $2^{\circ}$ Foundations of linear piezoelectricity. In: Parkus H, editor, Electromagnetic interaction in elastic solids. Wien, Springer, 1979, $3^{\circ}$ Mathematical models of phenomenological piezoelectricity. New problems in mechanics continua, Waterloo, Ontario: University of Waterloo Press, 1983, pp. 29 - 39.

Applications today: important roles in the design and health monitoring of ship and marine structures by establishing plentiful styles of devices such as sensors, actuators and power supplies with the responsibility of electro - magneto - mechanical energy conversion. Other fields of applications include: microwave electronics, optoelectronics, electronic instrumentation and scanning probe microscopy technique.

Due to exceptional functions of multifield materials such as flat frequency response and transformation of energy from one form to the other (mechanical, electric, magnetic or thermal energy) this type of composite exhibiting piezoelectric and piezomagnetic properties has found increasing applications in the following branches: aerospace, automotive, industries and submarines.

Nowadays, multifield composite materials have a wide range of applications in engineering science such as space planes, supersonic, air planes, rockets, missiles, fusion reactions and submarines.

This paper concerns some problems of mechanics of multifield materials. The results are new in the world science. I addresses it on the occasion of the $60^{\text {th }}$ anniversary of my Department and $50-y e a r s$ of my educational and research work. 


\section{Quo vadis mechanics?}

\section{BIMIMETING IN MATERIALS ENGINEERING}

People observed and investigated products of nature to use its constructions in technology. "Bimimeting is about separating from nature good projects. It presents the way from biology to the nature", J. F. V. Vincent, Bimimeting modeling, Phil. Trans., R., Soc., London B, 358 (2003), 1597.

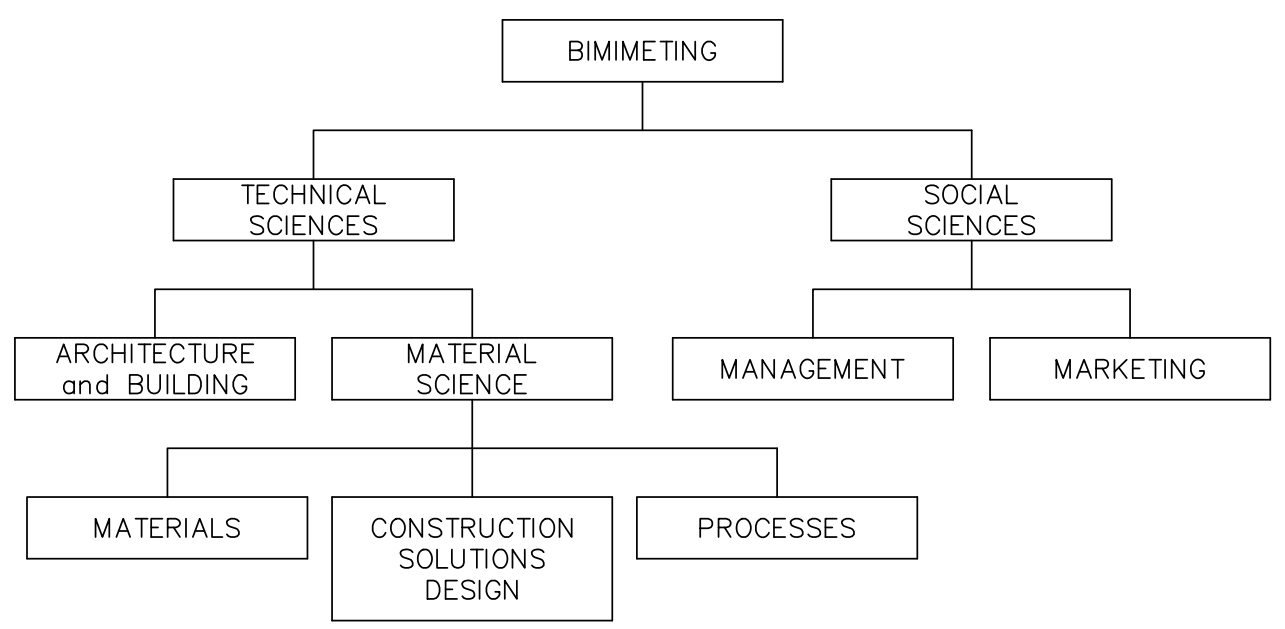

Fig.1. Bimimeting in dissimilar domains and disciplines of science (Konopka K., Wzorce $\mathrm{z}$ natury $\mathrm{w}$ technice i inżynierii materiałowej (Models from the nature in technology and material engineering), Oficyna Wydawnicza Politechniki Warszawskiej, Warszawa 2011).

\section{MEANDERS OF MULTIFIELD MATERIALS}
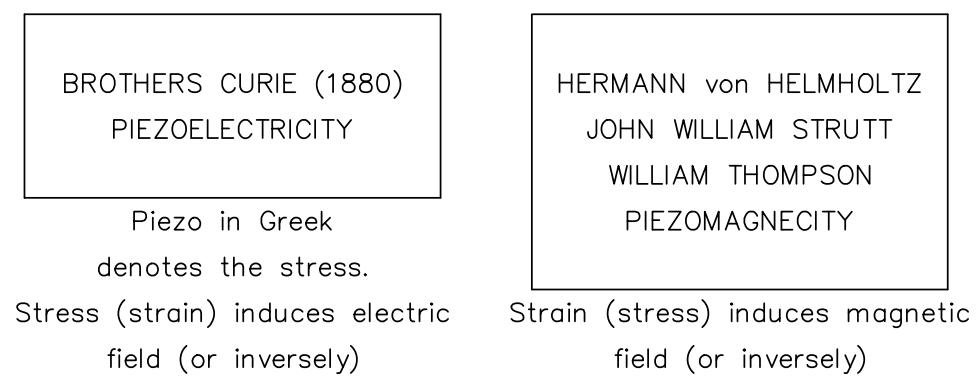

Def: Piezoelectricity is an electro - mechanical phenomenon, which couples elasticity and electricity through the existence of pressure induced electrical field or electric induced stress field. 


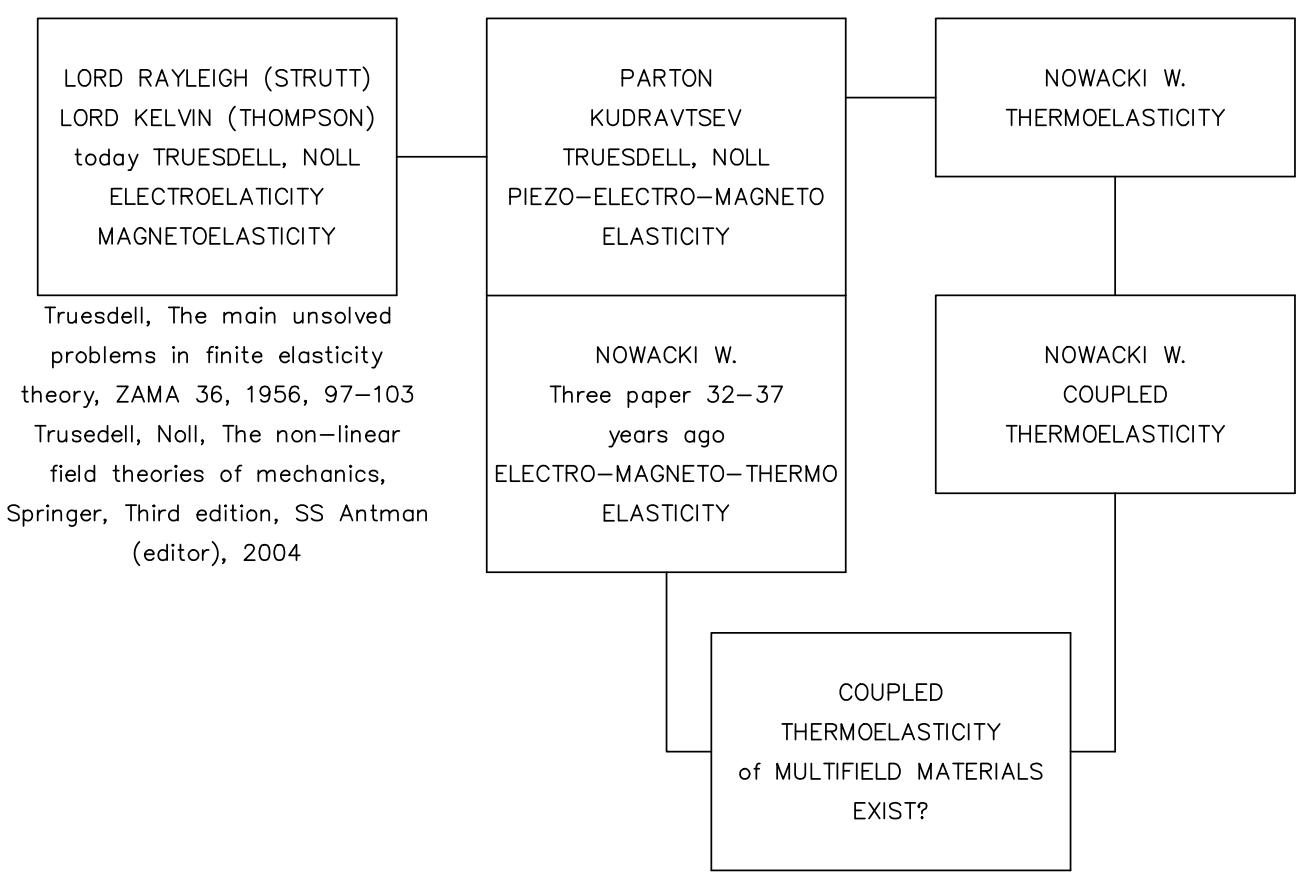

Thermoelasticity - If temperature of the body increases (decreases) the displacements change and the stresses occur.

Does inverse phenomenon exist?

$W$. Nowacki published some papers on coupled thermoelasticity. Other scientists in experiments did not accept this theory. I think that such materials exist in nature, so the change of displacement, electric and magnetic fields changes the temperature of the body. Does inverse coupled thermomagnetoelectroelasticity exist or not? The answer is as follows: It does in theory, but materials in nature have not been discovered yet.

\section{Appendix A. The material coefficients for mulifield materials}

A1. The material parameters in the characteristic Eq.(2.6) are as follows

$$
\begin{aligned}
& a=c_{44}\left[\mu_{33} e_{33}^{2}+\varepsilon_{33} q_{33}^{2}+c_{33} \mu_{33} \varepsilon_{33}-d_{33}\left(c_{33} d_{33}+2 e_{33} q_{33}\right)\right], \\
& b=\mu_{33}\left\{\left(e_{31}+e_{15}\right)\left[2 c_{13} e_{33}-c_{33}\left(e_{31}+e_{15}\right)\right]+2 c_{44} e_{33} e_{31}-c_{11} e_{33}^{2}-c_{33} c_{44} \varepsilon_{11}\right\}+ \\
& +\varepsilon_{33}\left\{\left(q_{31}+q_{15}\right)\left[2 c_{13} q_{33}-c_{33}\left(q_{31}+q_{15}\right)\right]+2 c_{44} q_{33} q_{31}-c_{11} q_{33}^{2}-c_{33} c_{44} \mu_{11}\right\}+ \\
& -\mu_{33} \varepsilon_{33} \tilde{c}^{2}-\left(e_{31}+e_{15}\right)^{2} q_{33}^{2}-\left(q_{31}+q_{15}\right)^{2} e_{33}^{2}-c_{44} \mu_{11} e_{33}^{2}-c_{44} \varepsilon_{11} q_{33}^{2}+ \\
& +2 e_{33} q_{33}\left(q_{31}+q_{15}\right)\left(e_{31}+e_{15}\right)+d_{33}^{2} \tilde{c}^{2}+2 c_{33} d_{33}\left(e_{31}+e_{15}\right)\left(q_{31}+q_{15}\right)+ \\
& +2 c_{44} c_{33} d_{11} d_{33}+2 e_{33} q_{33}\left(c_{44} d_{11}+c_{11} d_{33}\right)-2 d_{33}\left(c_{13}+c_{44}\right)\left[e_{33}\left(q_{31}+q_{15}\right)+q_{33}\left(e_{31}+e_{15}\right)\right],
\end{aligned}
$$




$$
\begin{aligned}
& c=\mu_{33}\left\{2 e_{15}\left[c_{11} e_{33}-c_{13}\left(e_{31}+e_{15}\right)\right]+c_{44} e_{31}^{2}+\varepsilon_{11} \tilde{c}^{2}\right\}+ \\
& +\varepsilon_{33}\left\{2 q_{15}\left[c_{11} q_{33}-c_{13}\left(q_{31}+q_{15}\right)\right]+c_{44} q_{31}^{2}+\mu_{11} \tilde{c}^{2}\right\}+ \\
& +c_{33} c_{44} \mu_{11} \varepsilon_{11}+c_{11} c_{44} \mu_{33} \varepsilon_{33}+2\left(c_{13}+c_{44}\right)\left(q_{31}+q_{15}\right)\left(d_{11} e_{33}+d_{33} e_{15}-q_{33} \varepsilon_{11}\right)+ \\
& +2\left(c_{13}+c_{44}\right)\left(e_{31}+e_{15}\right)\left(d_{11} q_{33}+d_{33} q_{15}-e_{33} \mu_{11}\right)+ \\
& +\left(q_{31}+q_{15}\right)^{2}\left(c_{33} \varepsilon_{11}+2 e_{33} e_{15}\right)+\left(e_{31}+e_{15}\right)^{2}\left(c_{33} \mu_{11}+2 q_{33} q_{15}\right)+ \\
& -2\left(q_{31}+q_{15}\right)\left(e_{31}+e_{15}\right)\left(e_{33} q_{15}+q_{33} e_{15}+c_{33} d_{11}+c_{44} d_{33}\right)+ \\
& -2 c_{11} d_{33}\left(e_{33} q_{15}+q_{33} e_{15}\right)-2 c_{44} d_{11}\left(q_{33} e_{15}+e_{33} q_{15}\right)+ \\
& -2 c_{11} d_{11} q_{33} e_{33}-2 c_{44} d_{33} q_{15} e_{15}+2 c_{44} q_{15} q_{33} \varepsilon_{11}+2 c_{44} e_{15} e_{33} \mu_{11}+ \\
& +c_{11} q_{33}^{2} \varepsilon_{11}+c_{11} e_{33}^{2} \mu_{11}-2 \tilde{c}^{2} d_{33} d_{11}-c_{11} c_{44} d_{33}^{2}-c_{44} c_{33} d_{11}^{2}, \\
& d=-c_{11} \mu_{33}\left(c_{44} \varepsilon_{11}+e_{15}^{2}\right)-c_{11} \varepsilon_{33}\left(c_{44} \mu_{11}+q_{15}^{2}\right)-c_{44}\left(e_{31}^{2} \mu_{11}+q_{31}^{2} \varepsilon_{11}\right)+ \\
& -e_{31}^{2} q_{15}^{2}-q_{31}^{2} e_{15}^{2}-\mu_{11} \varepsilon_{11} \tilde{c}^{2}+d_{11} \tilde{c}^{2}+2 c_{11} c_{44} d_{11} d_{33}+ \\
& +2 c_{13} q_{15} q_{31} \varepsilon_{11}+2 c_{13} e_{15} e_{31} \mu_{11}-2 c_{11} q_{15} q_{33} \varepsilon_{11}-2 c_{11} e_{15} e_{33} \mu_{11}+ \\
& +2 c_{13} q_{15}^{2} \varepsilon_{11}+2 c_{13} e_{15}^{2} \mu_{11}+2 e_{31} e_{15} q_{31} q_{15}+2 c_{11} e_{15} q_{15} d_{33}+ \\
& +d_{11}\left[-2 c_{13} e_{15}\left(q_{15}+q_{31}\right)-2 c_{13} q_{15}\left(e_{15}+e_{31}\right)\right]+d_{11}\left[2 c_{11}\left(e_{15} q_{33}+q_{15} e_{33}\right)+2 c_{44} e_{31} q_{31}\right] \\
& e=c_{11}\left[\mu_{11} e_{15}^{2}+\varepsilon_{11} q_{15}^{2}+c_{44} \varepsilon_{11} \mu_{11}-d_{11}\left(c_{44} d_{11}+2 e_{15} q_{15}\right)\right] \\
& \tilde{c}^{2}=c_{11} c_{33}-c_{13}\left(c_{13}+2 c_{44}\right) .
\end{aligned}
$$

A2. The parameters $a_{1}, b_{1}, c_{1}, d_{1}$ (define $a_{1 i}$ ), and $a_{2}, b_{2}, c_{2}, d_{2}$ in Eq.(2.7) are

$$
\begin{aligned}
& a_{1}=\beta_{1}\left[c_{33}\left(\varepsilon_{33} \mu_{33}-d_{33}^{2}\right)+\mu_{33} e_{33}^{2}+\varepsilon_{33} q_{33}^{2}-2 e_{33} d_{33} q_{33}\right]+ \\
& +\beta_{3}\left[-\left(c_{13}+c_{44}\right)\left(\varepsilon_{33} \mu_{33}-d_{33}^{2}\right)-\left(e_{31}+e_{15}\right)\left(\mu_{33} e_{33}-d_{33} q_{33}\right)-\left(q_{31}+q_{15}\right)\left(q_{33} \varepsilon_{33}-d_{33} e_{33}\right)\right]+ \\
& +\gamma_{3}\left[-\left(c_{13}+c_{44}\right)\left(d_{33} e_{33}-q_{33} \varepsilon_{33}\right)+\left(e_{31}+e_{15}\right)\left(d_{33} c_{33}+q_{33} e_{33}\right)-\left(q_{31}+q_{15}\right)\left(c_{33} \varepsilon_{33}+e_{33}^{2}\right)\right]+ \\
& +p_{3}\left[-\left(c_{13}+c_{44}\right)\left(d_{33} q_{33}-e_{33} \mu_{33}\right)+\left(q_{31}+q_{15}\right)\left(d_{33} c_{33}+q_{33} e_{33}\right)-\left(e_{31}+e_{15}\right)\left(c_{33} \mu_{33}+q_{33}^{2}\right)\right],
\end{aligned}
$$




$$
\begin{aligned}
& b_{1}=\beta_{1}\left[c_{33}\left(2 d_{11} d_{33}-\varepsilon_{33} \mu_{11}-\mu_{33} \varepsilon_{11}\right)+c_{44}\left(d_{33}^{2}-\varepsilon_{33} \mu_{33}\right)-\varepsilon_{11} q_{33}^{2}-\mu_{11} e_{33}^{2}+\right. \\
& \left.+2 d_{33}\left(e_{33} q_{15}+q_{33} e_{15}\right)+2 d_{11} e_{33} q_{33}-2 q_{15} q_{33} \varepsilon_{33}-2 e_{15} e_{33} \mu_{33}\right]+ \\
& +\beta_{3}\left[-\left(c_{13}+c_{44}\right)\left(2 d_{11} d_{33}-\varepsilon_{33} \mu_{11}-\mu_{33} \varepsilon_{11}\right)+\left(q_{13}+q_{15}\right)\left(q_{15} \varepsilon_{33}+q_{33} \varepsilon_{15}-d_{11} e_{33}-d_{33} e_{15}\right)+\right. \\
& \left.+\left(e_{31}+e_{15}\right)\left(e_{15} \mu_{33}+e_{33} \mu_{11}-d_{11} q_{33}-d_{11} q_{15}\right)\right]+ \\
& +\gamma_{3}\left[\left(c_{13}+c_{44}\right)\left(d_{11} e_{33}+d_{33} e_{15}-q_{15} \varepsilon_{33}-q_{33} \varepsilon_{11}\right)-\left(e_{31}+e_{15}\right)\left(c_{44} d_{33}+c_{33} d_{11}+q_{15} e_{33}+e_{15} q_{33}\right)+\right. \\
& \left.+\left(q_{31}+q_{15}\right)\left(c_{44} \varepsilon_{33}+c_{33} \varepsilon_{11}+2 e_{15} e_{33}\right)\right]+ \\
& +p_{3}\left[\left(c_{13}+c_{44}\right)\left(d_{11} q_{33}+d_{33} q_{15}-e_{15} \mu_{33}-e_{33} \mu_{11}\right)-\left(q_{31}+q_{15}\right)\left(c_{44} d_{33}+c_{33} d_{11}+q_{15} e_{33}+e_{15} q_{33}\right)+\right. \\
& \left.+\left(e_{31}+e_{15}\right)\left(c_{44} \mu_{33}+c_{33} \mu_{11}+2 q_{15} q_{33}\right)\right] \text {, } \\
& c_{1}=\beta_{1}\left[c_{44}\left(\varepsilon_{11} \mu_{33}+\varepsilon_{33} \mu_{11}-2 d_{11} d_{33}\right)+c_{33}\left(\varepsilon_{11} \mu_{11}-d_{11}^{2}\right)+\varepsilon_{33} q_{15}^{2}+\mu_{33} e_{15}^{2}+\right. \\
& \left.-2 d_{11}\left(e_{15} q_{33}+q_{15} e_{33}\right)+2 q_{15} q_{33} \varepsilon_{11}+2 \mu_{11} e_{15} e_{33}\right]+ \\
& +\beta_{3}\left[\left(c_{13}+c_{44}\right)\left(d_{11}^{2}-\varepsilon_{11} \mu_{11}\right)-\left(e_{31}+e_{15}\right)\left(\mu_{11} e_{15}-d_{11} q_{15}\right)-\left(q_{31}+q_{15}\right)\left(\varepsilon_{11} q_{15}-d_{11} e_{15}\right)\right]+ \\
& +\gamma_{3}\left[\left(c_{13}+c_{44}\right)\left(q_{15} \varepsilon_{11}-e_{15} d_{11}\right)+\left(e_{31}+e_{15}\right)\left(d_{11} c_{44}+q_{15} e_{15}\right)-\left(q_{31}+q_{15}\right)\left(c_{44} \varepsilon_{11}+e_{15}^{2}\right)\right]+ \\
& +p_{3}\left[\left(c_{13}+c_{44}\right)\left(e_{15} \mu_{11}-q_{15} d_{11}\right)+\left(q_{31}+q_{15}\right)\left(d_{11} c_{44}+q_{15} e_{15}\right)-\left(e_{31}+e_{15}\right)\left(c_{44} \mu_{11}+q_{15}^{2}\right)\right] \text {, } \\
& d_{1}=-\beta_{1}\left[c_{44}\left(\varepsilon_{11} \mu_{11}-d_{11}^{2}\right)+\mu_{11} e_{15}^{2}+\varepsilon_{11} q_{15}^{2}-2 e_{15} q_{15} d_{11}\right], \\
& a_{2}=c_{44}\left[\beta_{3}\left(\varepsilon_{33} \mu_{33}-d_{33}^{2}\right)+\gamma_{3}\left(d_{33} e_{33}+q_{33} \varepsilon_{33}\right)+p_{3}\left(d_{33} q_{33}-e_{33} \mu_{33}\right)\right], \\
& b_{2}=\beta_{1}\left[\left(c_{13}+c_{44}\right)\left(\varepsilon_{33} \mu_{33}-d_{33}^{2}\right)-\left(e_{31}+e_{15}\right)\left(d_{33} q_{33}-\mu_{33} e_{33}\right)-\left(q_{31}+q_{15}\right)\left(d_{33} e_{33}-\varepsilon_{33} q_{33}\right)\right]+ \\
& -\beta_{3}\left[c_{11}\left(\varepsilon_{33} \mu_{33}+d_{33}^{2}\right)+c_{44}\left(\mu_{11} \varepsilon_{33}+\mu_{33} \varepsilon_{11}\right)-2\left(q_{31}+q_{15}\right)\left(e_{31}+e_{15}\right) d_{33}+\right. \\
& \left.+\left(q_{31}+q_{15}\right)^{2} \varepsilon_{33}+\left(e_{31}+e_{15}\right)^{2} \mu_{33}\right]+ \\
& -\gamma_{3}\left[c_{11}\left(d_{33} e_{33}-q_{33} \varepsilon_{33}\right)+c_{44}\left(d_{11} e_{33}+d_{33} e_{15}-q_{15} \varepsilon_{33}-q_{33} \varepsilon_{11}\right)-\left(c_{13}+c_{44}\right) d_{33}\left(e_{31}+e_{15}\right)+\right. \\
& \left.-q_{33}\left(e_{31}+e_{15}\right)^{2}+\varepsilon_{33}\left(q_{31}+q_{15}\right)\left(c_{13}+c_{44}\right)+e_{33}\left(q_{31}+q_{15}\right)\left(e_{31}+e_{15}\right)\right]+ \\
& -p_{3}\left[c_{11}\left(d_{33} q_{33}-e_{33} \mu_{33}\right)+c_{44}\left(d_{11} q_{33}+d_{33} q_{15}-e_{15} \mu_{33}-e_{33} \mu_{11}\right)-\left(c_{13}+c_{44}\right) d_{33}\left(q_{31}+q_{15}\right)+\right. \\
& \left.-e_{33}\left(q_{31}+q_{15}\right)^{2}+\mu_{33}\left(e_{31}+e_{15}\right)\left(c_{13}+c_{44}\right)+q_{33}\left(q_{31}+q_{15}\right)\left(e_{31}+e_{15}\right)\right] \text {, }
\end{aligned}
$$




$$
\begin{aligned}
& c_{2}=\beta_{1}\left[-\left(c_{13}+c_{44}\right)\left(\varepsilon_{11} \mu_{33}+\varepsilon_{33} \mu_{11}\right)+\left(e_{31}+e_{15}\right)\left(d_{11} q_{33}+d_{33} q_{15}\right)+\left(q_{31}+q_{15}\right)\left(d_{11} e_{33}+d_{33} e_{15}\right)+\right. \\
& \left.-\left(q_{31}+q_{15}\right)\left(q_{15} \varepsilon_{33}+q_{33} \varepsilon_{11}\right)-\left(e_{31}+e_{15}\right)\left(e_{15} \mu_{33}+e_{33} \mu_{11}\right)+2\left(c_{13}+c_{44}\right) d_{11} d_{33}\right]+ \\
& +\beta_{3}\left[c_{44}\left(\varepsilon_{11} \mu_{11}+d_{11}^{2}\right)+c_{11}\left(\mu_{11} \varepsilon_{33}+\mu_{33} \varepsilon_{11}\right)+\mu_{11}\left(e_{31}+e_{15}\right)^{2}+\varepsilon_{11}\left(q_{31}+q_{15}\right)^{2}+\right. \\
& \left.-2\left(e_{31}+e_{15}\right)\left(q_{31}+q_{15}\right) d_{11}\right]+ \\
& -\gamma_{3}\left[c_{44}\left(q_{15} \varepsilon_{11}-e_{15} d_{11}\right)+\left(e_{31}+e_{15}\right)\left(\left(c_{13}+c_{44}\right) d_{11}+\left(e_{31}+e_{15}\right) q_{15}\right)+\right. \\
& \left.-\left(q_{31}+q_{15}\right)\left(\left(c_{13}+c_{44}\right) \varepsilon_{11}+\left(e_{31}+e_{15}\right) e_{15}\right)-c_{11}\left(d_{11} e_{33}+e_{15} d_{33}-\varepsilon_{11} q_{33}-\varepsilon_{33} q_{15}\right)\right]+ \\
& -p_{3}\left[c_{44}\left(e_{15} \mu_{11}-q_{15} d_{11}\right)+\left(q_{31}+q_{15}\right)\left(\left(c_{13}+c_{44}\right) d_{11}+\left(q_{31}+q_{15}\right) e_{15}\right)+\right. \\
& \left.-\left(e_{31}+e_{15}\right)\left(\left(c_{13}+c_{44}\right) \mu_{11}+\left(q_{31}+q_{15}\right) q_{15}\right)-c_{11}\left(d_{11} q_{33}+q_{15} d_{33}-\mu_{11} e_{33}-\mu_{33} e_{15}\right)\right], \\
& d_{2}=\beta_{1}\left[\left(c_{13}+c_{44}\right) \varepsilon_{11} \mu_{11}-\left(e_{31}+e_{15}\right) d_{11} q_{15}-\left(q_{31}+q_{15}\right) d_{11} e_{15}+\left(q_{31}+q_{15}\right) q_{15} \varepsilon_{11}+\right. \\
& \left.+\left(e_{31}+e_{15}\right) \mu_{11} e_{15}-\left(c_{13}+c_{44}\right) d_{11}^{2}\right]-\beta_{3}\left[c_{11}\left(\varepsilon_{11} \mu_{11}-d_{11}^{2}\right)\right]-\gamma_{3}\left[c_{11}\left(e_{15} d_{11}-q_{15} \varepsilon_{11}\right)\right]+ \\
& -p_{3}\left[c_{11}\left(q_{15} d_{11}-e_{15} \mu_{11}\right)\right] .
\end{aligned}
$$

A3. The parameters $a_{3 i}$ and $a_{4 i}$ in Eqs (2.1) are defined alternatively to those defined by Eqs (2.7) by parameter $a_{l i}$ as follows

$$
\begin{aligned}
& a_{3 i}=\left[\left\{\beta_{1}\left[\left(e_{31}+e_{15}\right)\left(q_{33} \lambda_{i}^{2}-q_{15}\right)-\left(c_{13}+c_{44}\right)\left(d_{11}-d_{33} \lambda_{i}^{2}\right)\right]+\right.\right. \\
& +\beta_{3}\left[\left(e_{31}+e_{15}\right)\left(q_{31}+q_{15}\right) \lambda_{i}+\left(c_{44} \lambda_{i}^{2}-c_{11}\right)\left(d_{11}-d_{33} \lambda_{i}^{2}\right)\right]+ \\
& \left.+p_{3}\left[\lambda_{i}\left(c_{13}+c_{44}\right)\left(q_{31}+q_{15}\right)+\left(c_{44} \lambda_{i}^{2}-c_{11}\right)\left(q_{33} \lambda_{i}^{2}-q_{15}\right)\right]\right\} a_{1 i} \lambda_{i}+ \\
& +\beta_{1}\left[\left(c_{33} \lambda_{i}^{2}-c_{44}\right)\left(d_{11}-d_{33} \lambda_{i}^{2}\right)-\left(q_{33} \lambda_{i}^{2}-q_{15}\right)\left(e_{33} \lambda_{i}^{2}-e_{15}\right)\right]+ \\
& +\beta_{3} \lambda_{i}\left[\left(c_{13}+c_{44}\right)\left(d_{11}-d_{33} \lambda_{i}^{2}\right)-\left(q_{31}+q_{15}\right)\left(e_{33} \lambda_{i}^{2}-e_{15}\right)\right]+ \\
& \left.+p_{3} \lambda_{i}\left[\left(c_{13}+c_{44}\right)\left(q_{33} \lambda_{i}^{2}-q_{15}\right)-\left(q_{31}+q_{15}\right)\left(c_{33} \lambda_{i}^{2}-c_{44}\right)\right]\right] \times \\
& \times\left\{p_{3} \lambda_{i}\left[\left(e_{31}+e_{15}\right)\left(q_{33} \lambda_{i}^{2}-q_{15}\right)-\left(q_{31}+q_{15}\right)\left(e_{33} \lambda_{i}^{2}-e_{15}\right)\right]+\right. \\
& +\beta_{1}\left[\left(e_{33} \lambda_{i}^{2}-e_{15}\right)\left(d_{11}-d_{33} \lambda_{i}^{2}\right)-\left(q_{33} \lambda_{i}^{2}-q_{15}\right)\left(\varepsilon_{11}-\varepsilon_{33} \lambda_{i}^{2}\right)\right]+ \\
& \left.+\beta_{3} \lambda_{i}\left[\left(e_{31}+e_{15}\right)\left(d_{11}-d_{33} \lambda_{i}^{2}\right)-\left(q_{31}+q_{15}\right)\left(\varepsilon_{11}-\varepsilon_{33} \lambda_{i}^{2}\right)\right]\right\}
\end{aligned}
$$




$$
\begin{aligned}
& a_{4 i}=-\left[\left\{\beta_{1}\left[\left(e_{31}+e_{15}\right)\left(e_{33} \lambda_{i}^{2}-e_{15}\right)-\left(c_{13}+c_{44}\right)\left(\varepsilon_{11}-\varepsilon_{33} \lambda_{i}^{2}\right)\right]+\right.\right. \\
& +\beta_{3}\left[\left(e_{31}+e_{15}\right)^{2} \lambda_{i}+\left(c_{44} \lambda_{i}^{2}-c_{11}\right)\left(\varepsilon_{11}-\varepsilon_{33} \lambda_{i}^{2}\right)\right]+ \\
& \left.+p_{3}\left[\lambda_{i}\left(c_{13}+c_{44}\right)\left(e_{31}+e_{15}\right)+\left(c_{44} \lambda_{i}^{2}-c_{11}\right)\left(e_{33} \lambda_{i}^{2}-e_{15}\right)\right]\right\} a_{1 i} \lambda_{i}+ \\
& +\beta_{1}\left[\left(c_{33} \lambda_{i}^{2}-c_{44}\right)\left(\varepsilon_{11}-\varepsilon_{33} \lambda_{i}^{2}\right)-\left(e_{33} \lambda_{i}^{2}-e_{15}\right)^{2}\right]+ \\
& +\beta_{3} \lambda_{i}\left[\left(c_{13}+c_{44}\right)\left(\varepsilon_{11}-\varepsilon_{33} \lambda_{i}^{2}\right)-\left(e_{31}+e_{15}\right)\left(e_{33} \lambda_{i}^{2}-e_{15}\right)\right]+ \\
& \left.+p_{3} \lambda_{i}\left[\left(c_{13}+c_{44}\right)\left(e_{33} \lambda_{i}^{2}-e_{15}\right)-\left(e_{31}+e_{15}\right)\left(c_{33} \lambda_{i}^{2}-c_{44}\right)\right]\right] \times \\
& \times\left\{p_{3} \lambda_{i}\left[\left(e_{31}+e_{15}\right)\left(q_{33} \lambda_{i}^{2}-q_{15}\right)-\left(q_{31}+q_{15}\right)\left(e_{33} \lambda_{i}^{2}-e_{15}\right)\right]+\right. \\
& +\beta_{1}\left[\left(e_{33} \lambda_{i}^{2}-e_{15}\right)\left(d_{11}-d_{33} \lambda_{i}^{2}\right)-\left(q_{33} \lambda_{i}^{2}-q_{15}\right)\left(\varepsilon_{11}-\varepsilon_{33} \lambda_{i}^{2}\right)\right]+ \\
& \left.+\beta_{3} \lambda_{i}\left[\left(e_{31}+e_{15}\right)\left(d_{11}-d_{33} \lambda_{i}^{2}\right)-\left(q_{31}+q_{15}\right)\left(\varepsilon_{11}-\varepsilon_{33} \lambda_{i}^{2}\right)\right]\right\}^{-1}
\end{aligned}
$$

A4. The roots of characteristic Eq.(2.6) are presented by formulae

$$
\begin{aligned}
& \lambda_{1}^{2}=-\frac{b}{4 a}-\frac{1}{2} \sqrt{R_{5}+R_{6}}-\frac{1}{2} \sqrt{2 R_{5}-R_{6}+\frac{1}{4} \frac{R_{7}}{\sqrt{R_{5}+R_{6}}}}, \\
& \lambda_{2}^{2}=-\frac{b}{4 a}-\frac{1}{2} \sqrt{R_{5}+R_{6}}+\frac{1}{2} \sqrt{2 R_{5}-R_{6}+\frac{1}{4} \frac{R_{7}}{\sqrt{R_{5}+R_{6}}}}, \\
& \lambda_{3}^{2}=-\frac{b}{4 a}+\frac{1}{2} \sqrt{R_{5}+R_{6}}-\frac{1}{2} \sqrt{2 R_{5}-R_{6}-\frac{1}{4} \frac{R_{7}}{\sqrt{R_{5}+R_{6}}}}, \\
& \lambda_{4}^{2}=-\frac{b}{4 a}+\frac{1}{2} \sqrt{R_{5}+R_{6}}+\frac{1}{2} \sqrt{2 R_{5}-R_{6}-\frac{1}{4} \frac{R_{7}}{\sqrt{R_{5}+R_{6}}}}
\end{aligned}
$$

where

$$
\begin{array}{ll}
R_{1}=2 c^{3}-9 b c d+27 a d^{2}+27 b^{2} e-72 a c e ; & R_{2}=c^{2}-3 b d+12 a e, \\
R_{3}=\sqrt{R_{1}^{2}-4 R_{2}^{3}} ; \quad R_{4}=\sqrt[3]{\frac{1}{2}\left(R_{1}+R_{3}\right)}, & \\
R_{5}=\frac{b^{2}}{4 a^{2}}-\frac{2 c}{3 a} ; \quad R_{6}=\frac{R_{2}}{3 a R_{4}}+\frac{R_{4}}{3 a} ; \quad R_{7}=\frac{b^{3}}{a^{3}}-\frac{4 b c}{a^{2}}+\frac{8 d}{a} .
\end{array}
$$


A5. The material parameters in Eqs (3.12) are as follows

$$
\begin{aligned}
& d_{1}^{*}=a_{32}\left(\frac{a_{44} a_{53}}{\lambda_{3}}-\frac{a_{43} a_{54}}{\lambda_{4}}\right)+a_{33}\left(\frac{a_{42} a_{54}}{\lambda_{4}}-\frac{a_{44} a_{52}}{\lambda_{2}}\right)+a_{34}\left(\frac{a_{43} a_{52}}{\lambda_{2}}-\frac{a_{42} a_{53}}{\lambda_{3}}\right) \\
& d_{2}^{*}=a_{31}\left(\frac{a_{43} a_{54}}{\lambda_{4}}-\frac{a_{44} a_{53}}{\lambda_{3}}\right)+a_{33}\left(\frac{a_{44} a_{51}}{\lambda_{1}}-\frac{a_{41} a_{54}}{\lambda_{4}}\right)+a_{34}\left(\frac{a_{41} a_{53}}{\lambda_{3}}-\frac{a_{43} a_{51}}{\lambda_{1}}\right) \\
& d_{3}^{*}=a_{31}\left(\frac{a_{44} a_{52}}{\lambda_{2}}-\frac{a_{42} a_{54}}{\lambda_{4}}\right)+a_{32}\left(\frac{a_{41} a_{54}}{\lambda_{4}}-\frac{a_{44} a_{51}}{\lambda_{1}}\right)+a_{34}\left(\frac{a_{42} a_{51}}{\lambda_{1}}-\frac{a_{41} a_{52}}{\lambda_{2}}\right) \\
& d_{4}^{*}=a_{31}\left(\frac{a_{42} a_{53}}{\lambda_{3}}-\frac{a_{43} a_{52}}{\lambda_{2}}\right)+a_{32}\left(\frac{a_{43} a_{51}}{\lambda_{1}}-\frac{a_{41} a_{53}}{\lambda_{3}}\right)+a_{33}\left(\frac{a_{41} a_{52}}{\lambda_{2}}-\frac{a_{42} a_{51}}{\lambda_{1}}\right) \\
& m_{2}^{*}=\sum_{i=1}^{4} a_{5 i} d_{i}^{*} .
\end{aligned}
$$

A6. The material parameters $d_{i}, l_{i}$, and $k_{i}$ in Eqs (5.8) and (5.11); $i=1,2,3,4$, are as follows

$$
\begin{aligned}
& d_{1}=a_{52}\left(a_{33} a_{44}-a_{43} a_{34}\right)+a_{53}\left(a_{34} a_{42}-a_{32} a_{44}\right)+a_{54}\left(a_{32} a_{43}-a_{33} a_{42}\right), \\
& d_{2}=a_{51}\left(a_{34} a_{43}-a_{33} a_{44}\right)+a_{53}\left(a_{31} a_{44}-a_{34} a_{41}\right)+a_{54}\left(a_{33} a_{41}-a_{31} a_{43}\right), \\
& d_{3}=a_{51}\left(a_{32} a_{44}-a_{34} a_{42}\right)+a_{52}\left(a_{34} a_{41}-a_{31} a_{44}\right)+a_{54}\left(a_{31} a_{42}-a_{32} a_{41}\right), \\
& d_{4}=a_{51}\left(a_{33} a_{42}-a_{32} a_{43}\right)+a_{52}\left(a_{31} a_{43}-a_{33} a_{41}\right)+a_{53}\left(a_{32} a_{41}-a_{31} a_{42}\right), \\
& l_{1}=a_{52}\left(a_{43}-a_{44}\right)+a_{53}\left(a_{44}-a_{42}\right)+a_{54}\left(a_{42}-a_{43}\right), \\
& l_{2}=a_{51}\left(a_{44}-a_{43}\right)+a_{53}\left(a_{41}-a_{44}\right)+a_{54}\left(a_{43}-a_{41}\right), \\
& l_{3}=a_{51}\left(a_{42}-a_{44}\right)+a_{52}\left(a_{44}-a_{41}\right)+a_{54}\left(a_{41}-a_{42}\right), \\
& l_{4}=a_{51}\left(a_{43}-a_{42}\right)+a_{52}\left(a_{41}-a_{43}\right)+a_{53}\left(a_{42}-a_{41}\right), \\
& k_{1}=a_{52}\left(a_{34}-a_{33}\right)+a_{53}\left(a_{32}-a_{34}\right)+a_{54}\left(a_{33}-a_{32}\right), \\
& k_{2}=a_{51}\left(a_{33}-a_{34}\right)+a_{53}\left(a_{34}-a_{31}\right)+a_{54}\left(a_{31}-a_{33}\right), \\
& k_{3}=a_{51}\left(a_{34}-a_{32}\right)+a_{52}\left(a_{31}-a_{34}\right)+a_{54}\left(a_{32}-a_{31}\right), \\
& k_{4}=a_{51}\left(a_{32}-a_{33}\right)+a_{52}\left(a_{33}-a_{31}\right)+a_{53}\left(a_{31}-a_{32}\right) .
\end{aligned}
$$


A7. The parameters in Eqs (4.6) have the following matrix forms

$$
\begin{aligned}
& \tilde{d}_{1}=-\left[\begin{array}{ccc}
a_{52} & a_{53} & a_{54} \\
a_{62} \lambda_{2} & a_{63} \lambda_{3} & a_{64} \lambda_{4} \\
a_{72} \lambda_{2} & a_{73} \lambda_{3} & a_{74} \lambda_{4}
\end{array}\right], \quad \tilde{d}_{2}=\left[\begin{array}{ccc}
a_{51} & a_{53} & a_{54} \\
a_{61} \lambda_{1} & a_{63} \lambda_{3} & a_{64} \lambda_{4} \\
a_{71} \lambda_{1} & a_{73} \lambda_{3} & a_{74} \lambda_{4}
\end{array}\right], \\
& \tilde{d}_{3}=-\left[\begin{array}{ccc}
a_{51} & a_{52} & a_{54} \\
a_{61} \lambda_{1} & a_{62} \lambda_{2} & a_{64} \lambda_{4} \\
a_{71} \lambda_{1} & a_{72} \lambda_{2} & a_{74} \lambda_{4}
\end{array}\right], \quad \tilde{d}_{4}=\left[\begin{array}{ccc}
a_{51} & a_{52} & a_{53} \\
a_{61} \lambda_{1} & a_{62} \lambda_{2} & a_{63} \lambda_{3} \\
a_{71} \lambda_{1} & a_{72} \lambda_{2} & a_{73} \lambda_{3}
\end{array}\right], \\
& \tilde{l}_{1}=\left[\begin{array}{ccc}
a_{52} & a_{53} & a_{54} \\
\frac{a_{52}}{\lambda_{2}} & \frac{a_{53}}{\lambda_{3}} & \frac{a_{54}}{\lambda_{4}} \\
a_{72} \lambda_{2} & a_{73} \lambda_{3} & a_{74} \lambda_{4}
\end{array}\right], \quad \tilde{l}_{2}=-\left[\begin{array}{ccc}
a_{51} & a_{53} & a_{54} \\
\frac{a_{51}}{\lambda_{1}} & \frac{a_{53}}{\lambda_{3}} & \frac{a_{54}}{\lambda_{4}} \\
a_{71} \lambda_{1} & a_{73} \lambda_{3} & a_{74} \lambda_{4}
\end{array}\right], \\
& \tilde{l}_{3}=\left[\begin{array}{ccc}
a_{51} & a_{52} & a_{54} \\
\frac{a_{51}}{\lambda_{1}} & \frac{a_{52}}{\lambda_{2}} & \frac{a_{54}}{\lambda_{4}} \\
a_{71} \lambda_{1} & a_{72} \lambda_{2} & a_{74} \lambda_{4}
\end{array}\right], \quad \tilde{l}_{4}=-\left[\begin{array}{ccc}
a_{51} & a_{52} & a_{53} \\
\frac{a_{51}}{\lambda_{1}} & \frac{a_{52}}{\lambda_{2}} & \frac{a_{53}}{\lambda_{3}} \\
a_{71} \lambda_{1} & a_{72} \lambda_{2} & a_{73} \lambda_{3}
\end{array}\right], \\
& \tilde{k}_{1}=-\left[\begin{array}{ccc}
a_{52} & a_{53} & a_{54} \\
\frac{a_{52}}{\lambda_{2}} & \frac{a_{53}}{\lambda_{3}} & \frac{a_{54}}{\lambda_{4}} \\
a_{62} \lambda_{2} & a_{63} \lambda_{3} & a_{64} \lambda_{4}
\end{array}\right], \quad \tilde{k}_{2}=\left[\begin{array}{ccc}
a_{51} & a_{53} & a_{54} \\
\frac{a_{51}}{\lambda_{1}} & \frac{a_{53}}{\lambda_{3}} & \frac{a_{54}}{\lambda_{4}} \\
a_{61} \lambda_{1} & a_{63} \lambda_{3} & a_{64} \lambda_{4}
\end{array}\right], \\
& \tilde{k}_{3}=-\left[\begin{array}{ccc}
a_{51} & a_{52} & a_{54} \\
\frac{a_{51}}{\lambda_{1}} & \frac{a_{52}}{\lambda_{2}} & \frac{a_{54}}{\lambda_{4}} \\
a_{61} \lambda_{1} & a_{62} \lambda_{2} & a_{64} \lambda_{4}
\end{array}\right], \quad \tilde{k}_{4}=\left[\begin{array}{ccc}
a_{51} & a_{52} & a_{53} \\
\frac{a_{51}}{\lambda_{1}} & \frac{a_{52}}{\lambda_{2}} & \frac{a_{53}}{\lambda_{3}} \\
a_{61} \lambda_{1} & a_{62} \lambda_{2} & a_{63} \lambda_{3}
\end{array}\right]
\end{aligned}
$$

A8. The following integrals are used

$$
\begin{aligned}
& \int_{0}^{\infty} \frac{d}{d \xi}\left(\frac{\sin \xi a}{\xi}\right) e^{-\xi \lambda_{i} z} J_{0}(r \xi) d \xi=-a n_{i}\left[1-\zeta_{i}\left(\frac{\pi}{2}-\tan ^{-1} \zeta_{i}\right)\right], \\
& \int_{0}^{\infty} \frac{d}{d \xi}\left(\frac{\sin \xi a}{\xi}\right) e^{-\xi \lambda_{i} z} J_{l}(r \xi) d \xi=-\frac{r}{2}\left(\frac{\pi}{2}-\tan ^{-1} \zeta_{i}-\frac{\zeta_{i}}{1+\zeta_{i}^{2}}\right), \\
& \int_{0}^{\infty} \xi \frac{d}{d \xi}\left(\frac{\sin \xi a}{\xi}\right) e^{-\xi \lambda_{i} z} J_{0}(r \xi) d \xi=-\frac{\pi}{2}+\tan ^{-1} \zeta_{i}+\frac{\zeta_{i}}{\zeta_{i}^{2}+\eta_{i}^{2}}
\end{aligned}
$$




$$
\begin{aligned}
& \int_{0}^{\infty} \xi \frac{d}{d \xi}\left(\frac{\sin \xi a}{\xi}\right) e^{-\xi \lambda_{i} z} J_{l}(r \xi) d \xi=-\frac{r}{a} \frac{\eta_{i}}{\left(1+\zeta_{i}^{2}\right)\left(\zeta_{i}^{2}+\eta_{i}^{2}\right)}, \\
& \int_{0}^{\infty} \frac{\sin \xi a}{\xi^{2}} e^{-\xi \lambda_{i} z} J_{0}(r \xi) d \xi=-\frac{a}{2} \ln \frac{1+\eta_{i}}{1-\eta_{i}}+a \eta_{i}\left[1-\zeta_{i}\left(\frac{\pi}{2}-\tan ^{-1} \zeta_{i}\right)\right], \\
& \int_{0}^{\infty} \frac{\sin \xi a}{\xi^{2}} e^{-\xi \lambda_{i} z} J_{l}(r \xi) d \xi=\frac{a^{2}}{r} \zeta_{i}\left(1-\eta_{i}\right)+\frac{r}{2}\left(\frac{\pi}{2}-\tan ^{-1} \zeta_{i}-\frac{\zeta_{i}}{1+\zeta_{i}^{2}}\right), \\
& \int_{0}^{\infty} \frac{\sin \xi a}{\xi} e^{-\xi \lambda_{i} z} J_{0}(r \xi) d \xi=\frac{\pi}{2}-\tan ^{-1} \zeta_{i}, \\
& \int_{0}^{\infty} \frac{\sin \xi a}{\xi} e^{-\xi \lambda_{i} z} J_{l}(r \xi) d \xi=\frac{a}{r}\left(1-\eta_{i}\right)
\end{aligned}
$$

where

$$
\begin{aligned}
& \zeta_{i}\left(r, z, a, \lambda_{i}\right)=\frac{1}{\sqrt{2} a} \sqrt{\sqrt{\left(r^{2}+\lambda_{i}^{2} z^{2}-a^{2}\right)^{2}+4 \lambda_{i}^{2} z^{2} a^{2}}+\left(r^{2}+\lambda_{i}^{2} z^{2}-a^{2}\right)}, \\
& \eta_{i}\left(r, z, a, \lambda_{i}\right)=\frac{1}{\sqrt{2} a} \sqrt{\sqrt{\left(r^{2}+\lambda_{i}^{2} z^{2}-a^{2}\right)^{2}+4 \lambda_{i}^{2} z^{2} a^{2}}-\left(r^{2}+\lambda_{i}^{2} z^{2}-a^{2}\right)}
\end{aligned}
$$

and $\lambda_{i}$ are the roots of Eq.(2.6) with positive real parts.

Note that the improper integrals (A.8) are new analytical results obtained by the author in his textbooks. In addition, the improper integrals (A.8) $)_{5}$ and (A.8) ${ }_{6}$ are not presented earlier. The integrals (A.8) are useful in other problems of mechanics, which may be solved with the use of the integral Hankel transformation and integral equation technique method.

Received: April 30, 2015

Revised: June 17, 2015 